\title{
Exosomal long noncoding RNA LNMAT2 promotes lymphatic metastasis in bladder cancer
}

\author{
Changhao Chen, ${ }^{1,2}$ Yuming Luo, ${ }^{2,3}$ Wang He, ${ }^{1,2}$ Yue Zhao, ${ }^{4}$ Yao Kong, ${ }^{5}$ Hongwei Liu, ${ }^{1,2}$ Guangzheng Zhong, ${ }^{1,2}$ Yuting Li, ${ }^{6}$ Jun Li, \\ Jian Huang, ${ }^{1,2}$ Rufu Chen, ${ }^{8}$ and Tianxin Lin ${ }^{1,2}$ \\ 'Department of Urology, Sun Yat-sen Memorial Hospital, Guangzhou, Guangdong, China. 'Guangdong Provincial Key Laboratory of Malignant Tumor Epigenetics and Gene Regulation, State Key Laboratory \\ of Oncology in South China, Sun Yat-sen Memorial Hospital, Guangzhou, Guangdong, China. 32Department of Pancreatobiliary Surgery, Sun Yat-sen Memorial Hospital, Guangzhou, Guangdong, China. \\ ${ }^{4}$ Department of Interventional Oncology, the First Affiliated Hospital, Sun Yat-sen University, Guangzhou, Guangdong, China. ${ }^{5}$ Department of Ultrasound and ${ }^{6}$ Department of Medical Oncology, Sun Yat-sen \\ Memorial Hospital, Guangzhou, Guangdong, China. 'Department of Biochemistry, Zhongshan School of Medicine, Sun Yat-sen University, Guangzhou, Guangdong, China. ${ }^{8}$ Department of Ceneral Surgery, \\ Guangdong Provincial People's Hospital, Guangdong Academy of Medical Sciences, Guangzhou, Guangdong, China.
}

\begin{abstract}
Patients with bladder cancer (BCa) with clinical lymph node (LN) metastasis have an extremely poor prognosis. VECF-C has been demonstrated to play vital roles in LN metastasis in BCa. However, approximately $20 \%$ of BCa with LN metastasis exhibits low VECF-C expression, suggesting a VECF-C-independent mechanism for LN metastasis of BCa. Herein, we demonstrate that BCa cell-secreted exosome-mediated lymphangiogenesis promoted LN metastasis in BCa in a VECF-Cindependent manner. We identified an exosomal long noncoding RNA (IncRNA), termed lymph node metastasis-associated transcript 2 (LNMAT2), that stimulated human lymphatic endothelial cell (HLEC) tube formation and migration in vitro and enhanced tumor lymphangiogenesis and LN metastasis in vivo. Mechanistically, LNMAT2 was loaded to BCa cellsecreted exosomes by directly interacting with heterogeneous nuclear ribonucleoprotein A2B1 (hnRNPA2B1). Subsequently, exosomal LNMAT2 was internalized by HLECs and epigenetically upregulated prospero homeobox 1 (PROX1) expression by recruitment of hnRNPA2B1 and increasing the H3K4 trimethylation level in the PROX1 promoter, ultimately resulting in lymphangiogenesis and lymphatic metastasis. Therefore, our findings highlight a VEGF-C-independent mechanism of exosomal IncRNA-mediated LN metastasis and identify LNMAT2 as a therapeutic target for LN metastasis in BCa.
\end{abstract}

\section{Introduction}

Bladder cancer (BCa), one of the most commonly diagnosed malignancies worldwide, is the leading cause of cancer-related death among men, with an estimated 550,000 new cases and 20,000 deaths in 2018 (1). The prognosis of patients with BCa is closely associated with the presence of lymph node (LN) metastasis, which decreases the 5 -year survival rate from $77.6 \%$ to $18.6 \%$ (2). Despite the overwhelming evidence for the role of $\mathrm{LN}$ metastasis in cancer, the definite molecular mechanism that triggers it in $\mathrm{BCa}$ remains unclear. We have previously demonstrated that VEGF-C produced by tumor cells (3) and tumor-associated macrophages (TAMs) (4) plays a crucial role in BCa lymphangiogenesis and LN metastasis. However, approximately $20 \%$ of BCa with LN metastasis has low VEGF-C expression $(5,6)$, which suggests the existence of a VEGF-C-independent mechanism for lymphangiogenesis and $\mathrm{LN}$ metastasis in $\mathrm{BCa}$.

Prospero homeobox 1 (PROX1) is essential for lymphatic vascular system formation, regulating endothelial cell differentiation and inducing the lymphatic budding and extension of lymphatic endothelial cells $(7,8)$. Prox1 $^{-/-}$mice lack a lymphatic vascular sys-

Authorship note: CC, Y. Luo, WH, and YZ contributed equally to this study. Conflict of interest: The authors have declared that no conflict of interest exists. Copyright: () 2020, American Society for Clinical Investigation.

Submitted: June 6, 2019; Accepted: October 2, 2019; Published: December 3, 2019

Reference information: / Clin Invest. 2020;130(1):404-421.

https://doi.org/10.1172/JCl130892. tem and die within a few days of birth (9). PROX1 enhanced lymphatic endothelial cell proliferation through synergistic interaction with p50 to activate VEGFR3 expression (10). Nevertheless, the precise mechanism of cancer cell promotion of lymphangiogenesis and PROX1 upregulation remains unclear.

Exosomes are small microvesicles ranging from $30-150 \mathrm{~nm}$ in size that contain various types of nucleic acids, including mRNAs, miRNAs, and long noncoding RNAs (lncRNAs) (11, 12). Recently, cancer cell-secreted exosomes were identified as crucial messengers in intercellular communication associated with cancer-induced vascular permeability (13), inflammation (14), and bone marrow progenitor cell recruitment in distant organs (15). For example, EGFR-containing exosomes regulate the liver microenvironment to promote liver metastasis (16), exosomal $m i R-25-3 p$ participates in premetastatic niche formation (17), and exosomal miR-1247-3p facilitates lung metastasis by activating cancer-associated fibroblasts (18). However, the mechanism of cancer cell-secreted exosome regulation of lymphatic vascular system formation via the induction of lymphangiogenesis remains unknown, warranting further exploration.

Herein, we report that an lncRNA, LINCO0858, termed lymph node metastasis-associated transcript 2 (LNMAT2), was overexpressed in $\mathrm{BC}$ a tissues and highly enriched in urinary exosomes (urinary-EXO) from patients with $\mathrm{BCa}$, and correlated positively with LN metastasis. Exosomal LNMAT2 promoted lymphangiogenesis and LN metastasis in vitro and in vivo. Mechanistically, LNMAT2 was loaded to exosomes by direct interaction with heterogeneous 
A

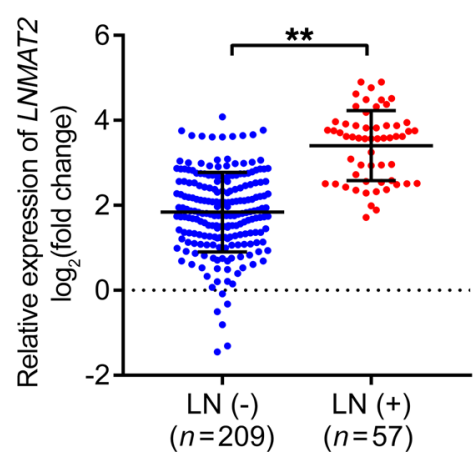

B

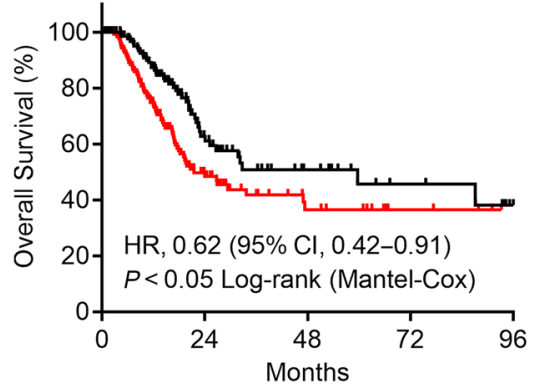

- Low LNMAT2 $(n=133)$

- High LNMAT2 $(n=133)$

$\mathrm{BCa}$

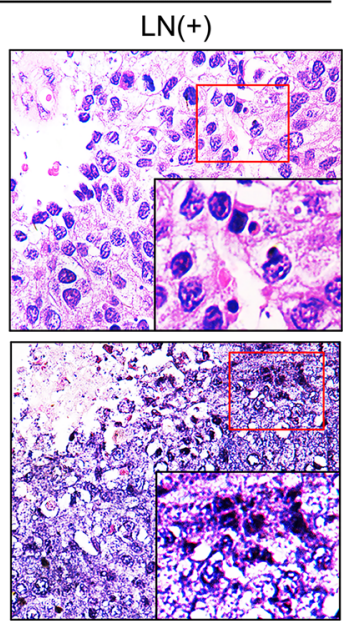

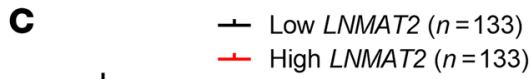

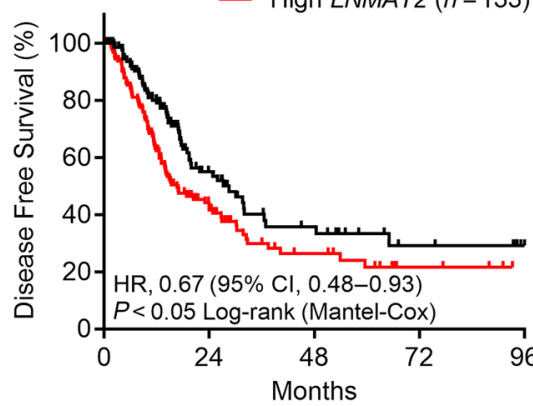

E

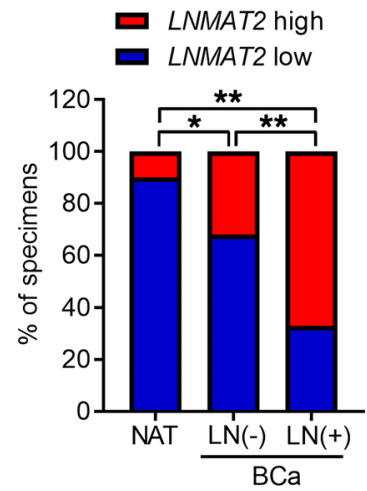

$\mathbf{F}$

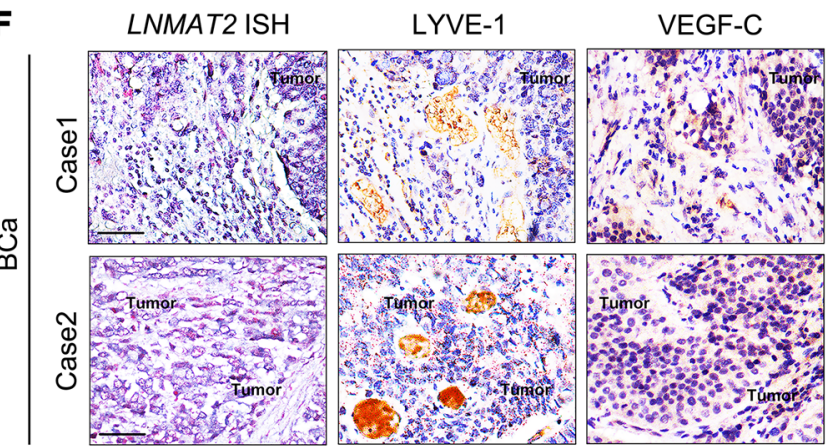

G

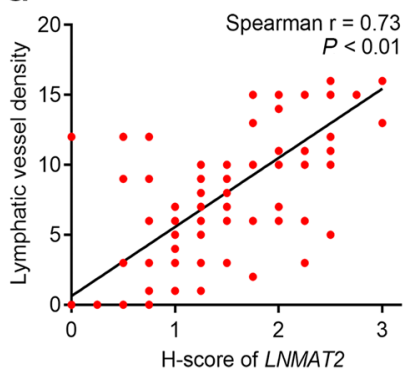

H

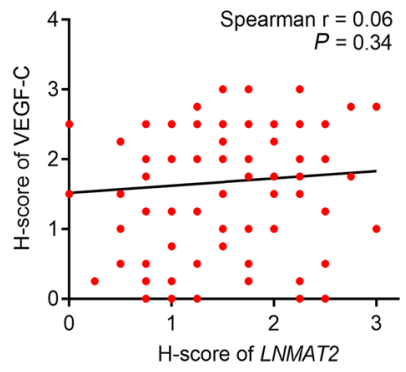

Figure 1. LNMAT2 overexpression is associated with BCa lymphatic metastasis. (A) qRT-PCR analysis of LNMAT2 expression in a cohort of $266 \mathrm{BCa}$ patients according to LN status. Groups were compared using the nonparametric Mann-Whitney $U$ test. GAPDH was used as an internal control. The OS (B) and DFS (C) of patients with BCa with lower vs. higher LNMAT2 expression were estimated using Kaplan-Meier curves. The median expression was used as the cut-off value. Representative ISH images (D) and percentages (E) of LNMAT2 expression (blue) in paraffin-embedded NAT and BCa tissue with or without LN metastasis $(n=266)$. Scale bars: $50 \mu \mathrm{m}$. Statistical significance was assessed by $\chi^{2}$ test. Representative images $(\mathbf{F})$ and correlation analysis $(\mathbf{G}$ and $\mathbf{H}$ ) of ISH and IHC staining showing positive correlation between LNMAT2 expression and lymphatic vessel density indicated by anti-LYVE-1 staining, and that LNMAT2 expression was not correlated with VEGF-C levels in the BCa tissues $(n=266)$. Scale bars: $50 \mu \mathrm{m} .{ }^{*} P<0.05 ;{ }^{* *} P<0.01$.

nuclear ribonucleoprotein A2B1 (hnRNPA2B1) and transmitted to human lymphatic endothelial cells (HLECs). Subsequently, LNMAT2 formed a triplex with the PROX1 promoter and enhanced PROX1 transcription by inducing hnRNPA2B1-mediated H3 lysine 4 trimethylation (H3K4me3), facilitating lymphangiogenesis and LN metastasis in BCa. Our findings highlight a VEGF-C-independent mechanism of exosomal LNMAT2-mediated LN metastasis and identify LNMAT2 as a potential diagnostic marker and therapeutic target for LN metastasis in BCa.

\section{Results}

LNMAT2 overexpression correlated with BCa LN metastasis. Using next-generation sequencing (NGS), we previously explored the global expression profiles of lncRNAs in high-grade muscleinvasive bladder cancer (MIBC) tissues and paired normal adjacent tissues (NATs) from 5 patients with BCa and in 5 paired LN-positive and LN-negative BCa tissues (4) (Gene Expression Omnibus ID GSE106534). Statistical analysis revealed that LNMAT2 expression was increased by more than 3 -fold in the 
MIBC tissues compared with the NATs and in the LN-positive $\mathrm{BCa}$ tissues compared with the LN-negative tissues. Quantitative real-time PCR (qRT-PCR) confirmed LNMAT2 overexpression in $\mathrm{BCa}$ tissues from 266 patients compared with the corresponding NATs (Supplemental Figure 1A; supplemental material available online with this article; https://doi. org/10.1172/JCI130892DS1). In humans, LNMAT2 is located on chromosome 10q23.1 (Ref-Seq accession number MK692948, Supplemental Figure 1B), and the full-length $3187 \mathrm{nt}$ LNMAT2 in $\mathrm{BCa}$ cells was determined by 5 ' and 3 'rapid amplification of cDNA ends (RACE) assays (Supplemental Figure 1, C-F). FISH and subcellular fractionation assays showed that LNMAT2 mainly localized to BCa cell cytoplasm (Supplemental Figure 2, A-D). Consistently, analyses of The Cancer Genome Atlas (TCGA) database showed that LNMAT2 was upregulated in multiple human cancers, such as $\mathrm{BCa}$, uterine corpus endometrial cancer, lung cancer, liver cancer, and stomach cancer (Supplemental Figure 3, A-F).

Moreover, a positive correlation was found between LNMAT2 expression and LN metastasis in a cohort of 266 BCa patients (Figure 1A and Supplemental Table 1). qRT-PCR detected higher LNMAT2 expression in metastatic tumor cells in the LNs than in $\mathrm{BCa}$ primary tumors, suggesting that LNMAT2 might contribute to BCa metastasis (Supplemental Figure 4A). Furthermore, Kaplan-Meier analysis revealed that LNMAT2 overexpression correlated with shorter overall survival (OS) and disease-free survival (DFS) in BCa patients (Figure 1, B and C). Univariate and multivariate Cox analysis confirmed that LNMAT2 expression correlated independently with OS and DFS in BCa patients (Supplemental Tables 2 and 3). Consistently, the TCGA database results indicated a positive association between LNMAT2 overexpression and poor prognosis in human cancer, including lung cancer and stomach cancer (Supplemental Figure 4, B-D). It is worth noting that LNMAT2 overexpression was highly correlated with reduced OS and DFS in LN-positive BCa patients (Supplemental Figure 4, E and $\mathrm{F}$ ). LNMAT2 expression was significantly upregulated in the LN-positive BCa tissues, slightly increased in LN-negative BCa tissues, and was rarely detected in NATs by in situ hybridization (ISH) assay (Figure 1, D and E, and Supplemental Figure 4G). Importantly, LNMAT2 expression was also positively correlated with lymphatic vessel density, as indicated by the specific lymphatic vessel marker lymphatic vessel endothelial hyaluronan receptor 1 (LYVE-1), but no correlation was observed between LNMAT2 and VEGF-C expression in BCa (Figure 1, F-H). Collectively, these results indicate that $L N M A T 2$ overexpression-induced LN metastasis of BCa might be independent of VEGF-C.

LNMAT2 was enriched in BCa cell-secreted exosomes. Prominently, ISH showed high LNMAT2 expression in the extracellular space of BCa (Figure 1D). We also found significantly higher extracellular LNMAT2 expression in BCa tissues with LN metastasis compared with $\mathrm{BCa}$ tissues without LN metastasis (Figure 1, D and E), suggesting that extracellular LNMAT2 might play a crucial role in BCa LN metastasis. As extracellular lncRNAs are mainly encapsulated in specific subcellular materials, exosomes in particular, and have important biological functions in mediating cell-cell interactions and contributing to tumor LN metastasis (19), we examined LNMAT2 expression in BCa cell-secreted exosomes. We used qRT-PCR to analyze the exosomes isolated from the urine samples of 206 patients with BCa and 120 healthy controls and found that LNMAT2 was upregulated in the urinary-EXO obtained from $\mathrm{BCa}$ patients compared with the healthy controls (Figure 2A), suggesting that exosomal LNMAT2 is essential to the development of BCa.

We further examined LNMAT2 expression in exosomes isolated from the culture medium of BCa cell lines (5637 and UM-UC-3). Exosomes with a typical cup-shaped morphology and $30-150 \mathrm{~nm}$ in size were detected by transmission electron microscopy (TEM) (Figure 2B and Supplemental Figure 4H) and NanoSight analysis (Figure 2C and Supplemental Figure 4I). Western blot detection of the exosomal protein markers CD9 and CD63 confirmed that the particles isolated from the culture medium were exosomes (Figure 2D and Supplemental Figure 4J). LNMAT2 expression was significantly upregulated in both the 5637 and UM-UC-3 cells and their corresponding exosomes as compared with that in human normal bladder epithelial cells (SV-HUC-1) (Figure 2E). Interestingly, LNMAT2 enrichment was detected in $\mathrm{BCa}$ cell-secreted exosomes relative to its expression in the cells, suggesting that LNMAT2 may exert its main function in exosomal form (Figure 2E). LNMAT2 overexpression via $L N M A T 2$ plasmid transfection led to an obvious increase of LNMAT2 levels in the BCa cell-secreted exosomes (Figure 2, F and $\mathrm{G}$ ), while silencing LNMAT2 had the opposite effect (Figure 2, $\mathrm{H}$ and I), suggesting that altering cellular LNMAT2 expression markedly impacts exosomal LNMAT2 expression, solidifying our hypothesis that extracellular LNMAT2 exists mainly in exosomal form. Taken together, our results indicate that LNMAT2 is abundant in BCa cell-secreted exosomes.

$B C$ a cell-secreted exosomal LNMAT2 promoted lymphangiogenesis in vitro. Since lymphangiogenesis is the rate-determining step for LN metastasis in BCa (20), we investigated whether upregulating LNMAT2 expression could facilitate lymphangiogenesis in vitro. The tube formation and migration in HLECs incubated with BCa cell-secreted exosomes were analyzed. The BCa cell-secreted exosomes dramatically promoted HLEC tube formation and migration as compared with the control (Figure 3, A-C). Moreover, the exosomes secreted by LNMAT2-overexpressing UM-UC-3 cells (UM-UC-3- $\mathrm{EXO}_{L N M A T 2}$ ) strongly induced HLEC tube formation and migration (Figure 3, D-F). Conversely, exosomes secreted by LNMAT2-silenced 5637 cells $\left(5637-\mathrm{EXO}_{\text {si-LNMAT2 }}\right.$ ) lost the ability to induce HLEC tube formation and migration (Figure 3, G-I). These results indicate that exosomal LNMAT2 contributes to lymphangiogenesis in vitro.

Exosomal LNMAT2 promoted lymphatic metastasis in vivo. Although we observed that LNMAT2 knockdown led to a reduction and LNMAT2 overexpression led to an increase in the growth rates of $\mathrm{BCa}$ cells, as indicated by CCK-8, colony formation, and EdU assays (Supplemental Figure 5, A-H), dysregulation of LNMAT2 has no obvious effect on the invasiveness of $\mathrm{BCa}$ cells, which provided further evidence that exosomal LNMAT2 plays vital roles in LN metastasis.

To further examine the effect of exosomal LNMAT2 on LN metastasis, we established a popliteal lymphatic metastasis model using similar modeling approaches as described previously $(4,21)$. Luciferase-labeled UM-UC-3 cells were implanted in the 
A

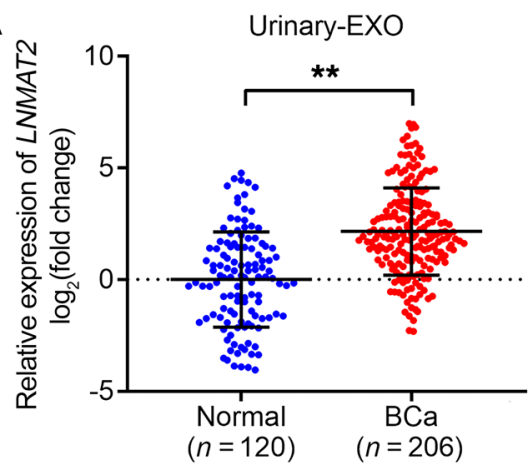

B

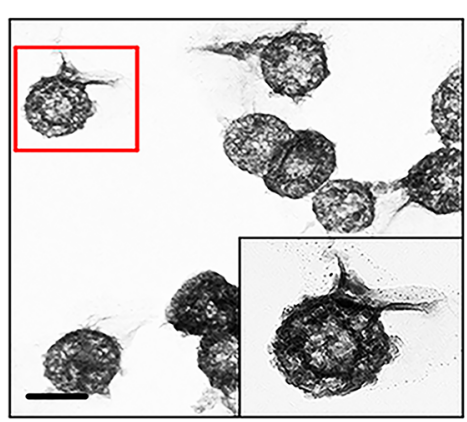

C

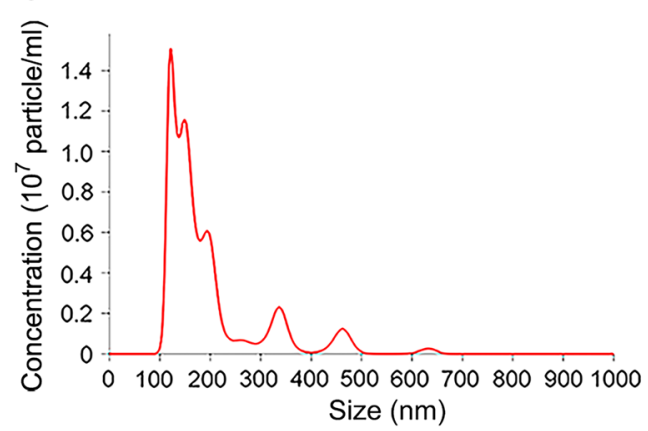

D

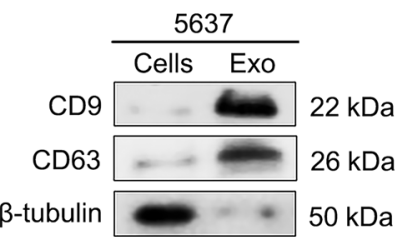

E

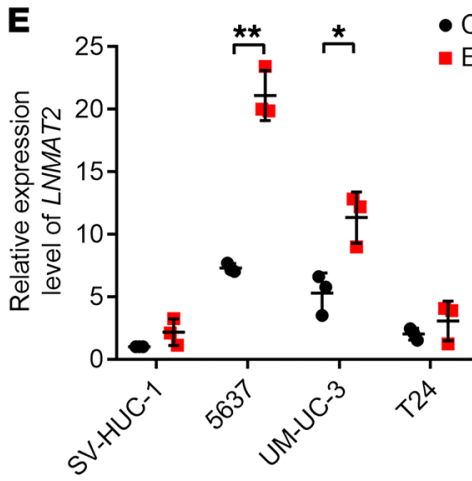

H

- Vector

- LNMAT2

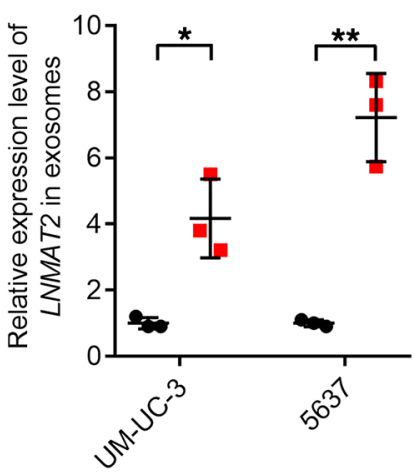

H $\quad$ si-NC

- si-LNMAT2\#1

- si-LNMAT2\#2

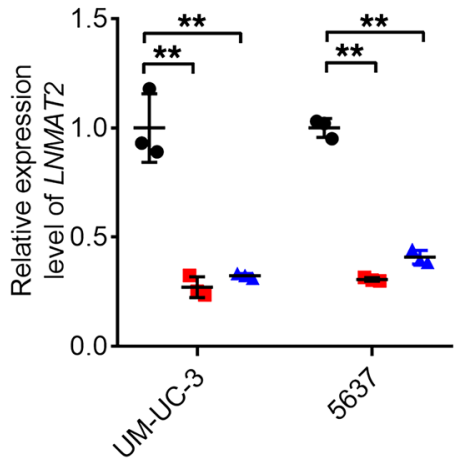

- Cellular F

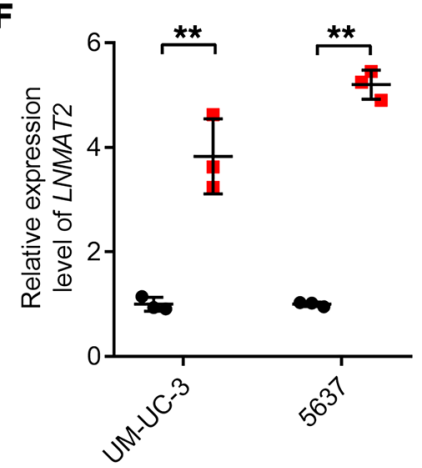

I

- si-NC

- si-LNMAT2\#1

- si-LNMAT2\#2

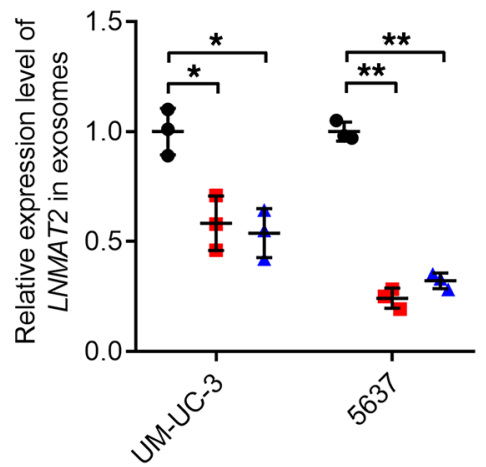

Figure 2. LNMAT2 is upregulated in BCa cell-secreted exosomes. (A) qRT-PCR analysis of LNMAT2 expression in urinary-EXO from 206 patients with $\mathrm{BCa}$ and 120 healthy participants. GAPDH was used as an internal control. Groups were compared using the nonparametric Mann-Whitney $U$ test. Purified 5637-EXO was identified by TEM (B) and NanoSight (C). Scale bars: $100 \mathrm{~nm}$. (D) Western blot analysis of exosomal protein markers in 5637 cell lysates or 5637-EXO. (E) qRT-PCR analysis of LNMAT2 expression levels in bladder cell lines and in their corresponding exosomes. GAPDH was used as an internal control. Statistical significance was assessed using 1-way ANOVA followed by Dunnett's tests. qRT-PCR analysis of LNMAT2 expression in LNMAT2-overexpressing control BCa cells $(\mathbf{F})$ and their corresponding exosomes (G). GAPDH was used as an internal control. Statistical significance was assessed using 2-tailed Student's $t$ test followed by Dunnett's tests for multiple comparisons. qRT-PCR detection of LNMAT2 expression in LNMAT2 knockdown control $\mathrm{BCa}$ cells $(\mathbf{H})$ and their corresponding exosomes (I). GAPDH was used as an internal control. Statistical significance was assessed using 1-way ANOVA followed by Dunnett's tests for multiple comparisons. Error bars represent the SD of 3 independent experiments. ${ }^{*} P<0.05 ;{ }^{* *} P<0.01$.

footpads of nude mice, which were then randomly divided into 3 groups $(n=12)$, followed by intratumoral injection with PBS (control), exosomes secreted by vector-transfected UM-UC-3 cells (UM-UC-3- $\left.\mathrm{EXO}_{\text {vector }}\right)$, or exosomes secreted by LNMAT2-transfected UM-UC-3 cells (UM-UC-3-EXO LNMAT2 ) every 3 days. When the primary tumor reached $200 \mathrm{~mm}^{3}$, the tumors and popliteal LNs were excised (Figure 4A). Interestingly, the live IVIS Spectrum In Vivo Imaging System showed that UM-UC-3-EXO $\mathrm{ENMAT2}_{\text {INA }}$ significantly promoted the ability of BCa cells to metastasize to the LNs as compared with the control or UM-UC-3-EXO groups (Figure 4, B and C). The volume of popliteal LNs in the $\mathrm{UM}-\mathrm{UC}-3-\mathrm{EXO}_{\text {LNMAT2 }}$ group was significantly larger than that in the control or UM-UC-3-EXO ${ }_{\text {vector }}$ groups (Figure 4, D and E). Luciferase immunostaining indicated increased metastatic LNs in

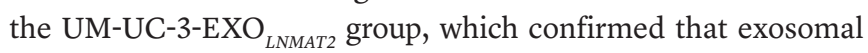
LNMAT2 significantly enhanced the metastatic capability of $\mathrm{BCa}$ 

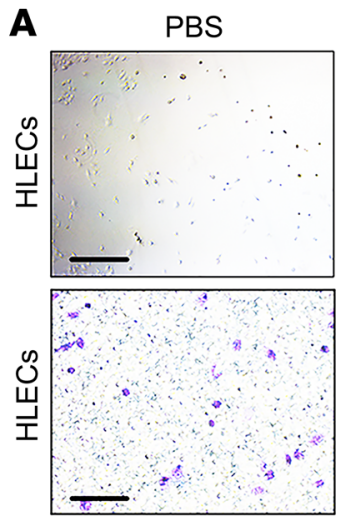

D UM-UC-3-EXOVector

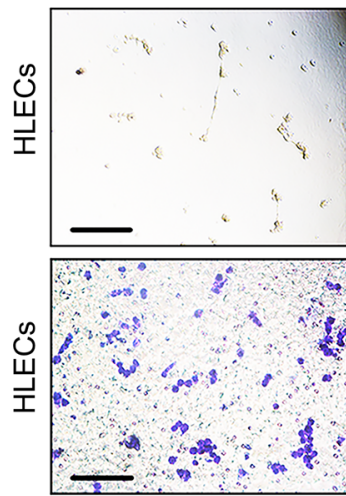

UM-UC-3-EXO
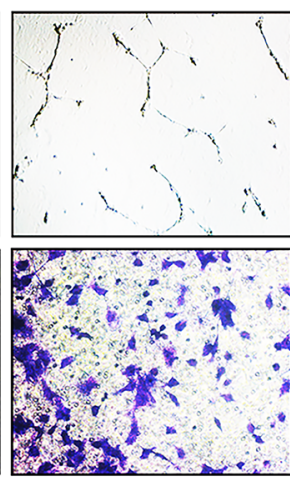

5637-EXO

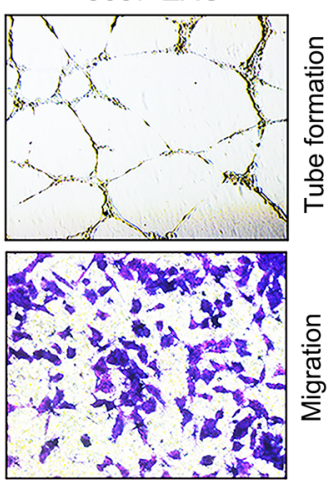

B

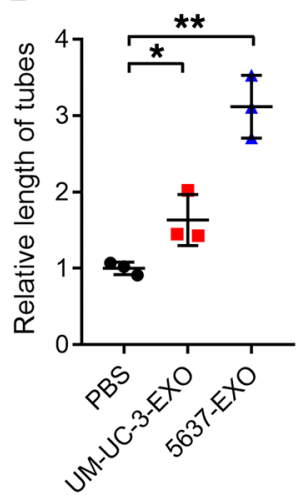

C

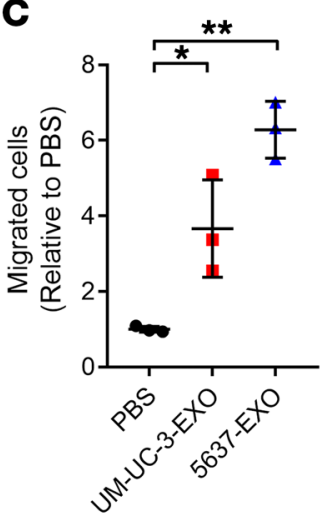

UM-UC-3-EXOLNMAT2

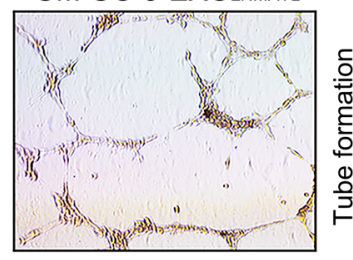

E

- UM-UC-3-EXO Vector

- UM-UC-3-EXO

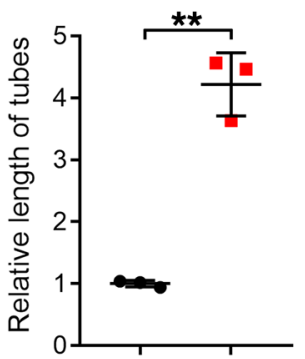

0
$\mathbf{F}$

- UM-UC-3-EXO Vector

- UM-UC-3-EXO

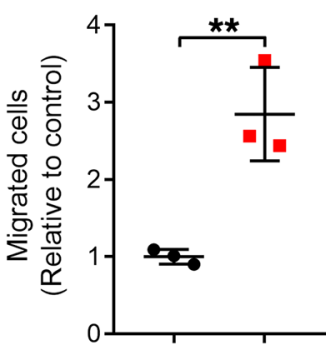

G

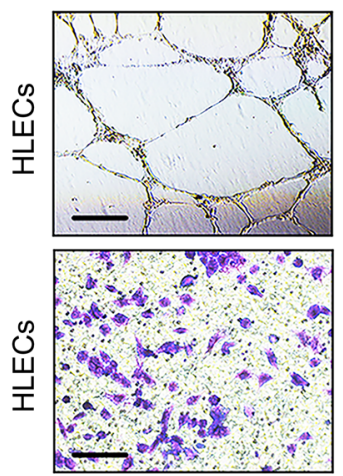

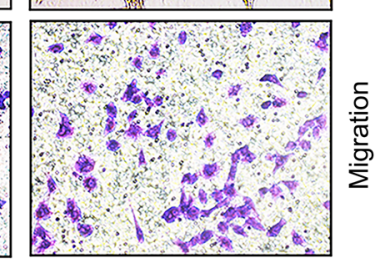

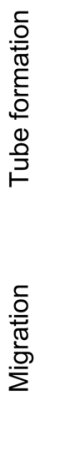

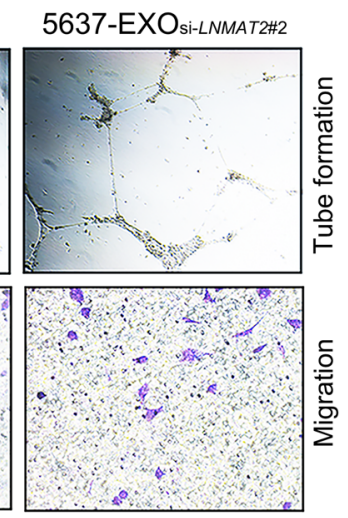

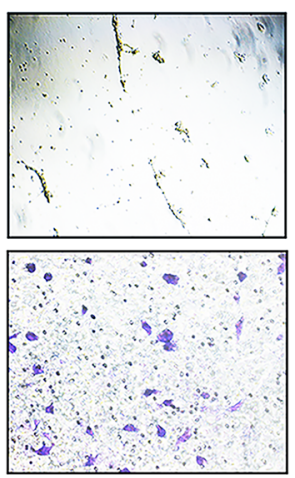

H

- 5637-EXO

- 5637-EXO si-LNMAT2\#1 $_{1}$

- 5637-EXO si-LNMAT2\#2 $^{2}$

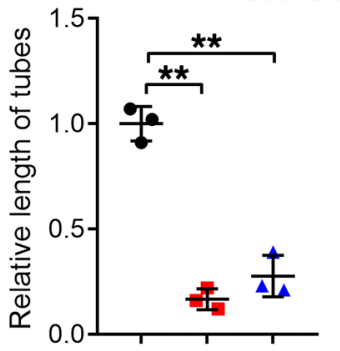

I
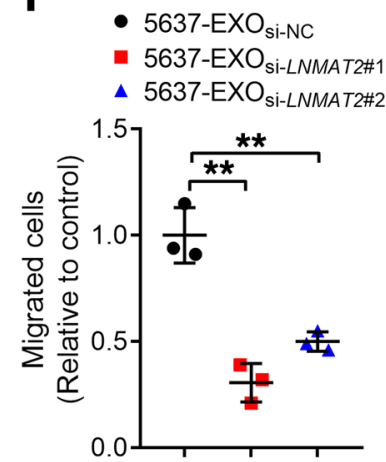

Figure 3. Exosomal LNMAT2 promotes lymphangiogenesis in vitro. Representative images (A) and quantification of tube formation (B) and Transwell migration (C) by HLECs treated with PBS, 5637-EXO, or UM-UC-3-EXO. Scale bars: $100 \mu \mathrm{m}$. Statistical significance was assessed using 1-way ANOVA followed by Dunnett's tests. Representative images (D) and quantification of tube formation (E) and Transwell migration (F) by HLECs treated with UM-UC-3-EXO Vector $_{\text {or UM-UC-3-EXO }}$ LNMAT2. Scale bars: $100 \mu \mathrm{m}$. Statistical significance was assessed using 2-tailed Student's $t$ test. Representative images (C) and quantification of tube formation (H) and Transwell migration (I) by HLECs treated with 5637-EXO ${\text { si-NC, } 5637-\mathrm{EXO}_{\text {si-LNMAT2\#11 }} \text {, or 5637-EXO }}_{\text {si-LNMAT2\#2. }}$. Scale bars: $100 \mu \mathrm{m}$. Statistical significance was assessed using 1-way ANOVA followed by Dunnett's tests. Error bars represent the SD of 3 independent experiments. ${ }^{*} P<0.05 ;{ }^{*} P<0.01$.

cells (Figure 4, F and G, and Supplemental Table 4). Taken together, these results suggest that exosomal LNMAT2 plays an important part in LN metastasis of BCa in vivo.

Tumorigenicity is a major factor underlying lymphangiogenesis and LN metastasis (22), and is closely associated with LN involvement in various solid tumors, such as lung cancer (23), gastric cancer (24), and appendix neuroendocrine tumor (25). Therefore, we investigated the tumorigenic capacity of exosomal LNMAT2 in vivo using a subcutaneous xenograft model as previously reported $(3,21)$. Mice were inoculated subcutaneously with UM-UC-3 cells and randomly separated into 3 groups $(n=12)$. Each group received intratumoral PBS, UM-UC-3-EXO ${ }_{\text {Vector }}$, or UM-UC-3-EXO ${ }_{L N M A T 2}$ every 3 days for 5 consecutive weeks (Figure 5A). UM-UC-3-EXO $\mathrm{EXNMAT2}_{\text {enhanced }}$ tumor growth compared with both the UM-UC-3-EXO Vector $_{\text {group and }}$ the control group (Figure 5, B-D). Tumors in the UM-UC-3-EXO $\mathrm{ENMAT2}_{2}$ group were of greater size and weight (Figure 5, E and F) and had 
A

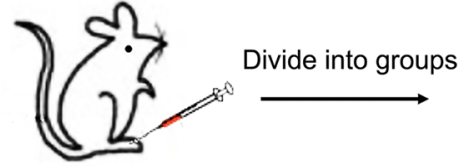

Inoculated with UM-UC-3 into footpad

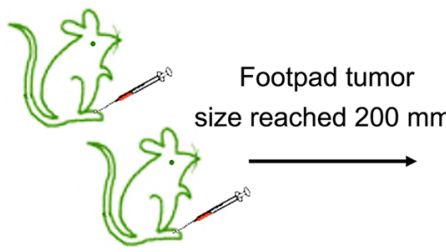

Exosomes intratumor injection

(Every 3 days)
B

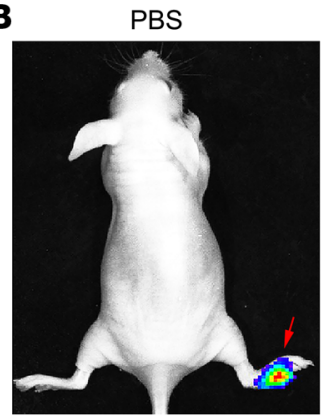

D

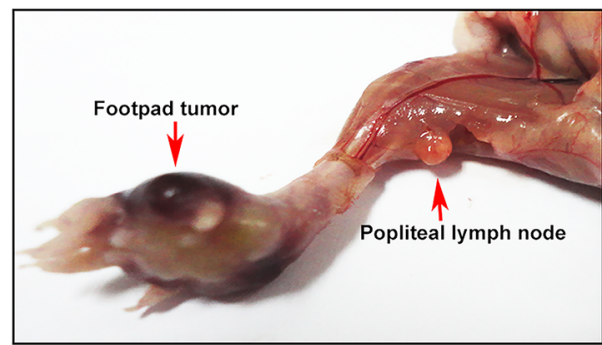

$F$
$F$
0
0
$\frac{1}{0}$
0
$\frac{0}{0}$
$\frac{0}{0}$
$\frac{10}{1}$
$\frac{1}{2}$
$\frac{1}{4}$

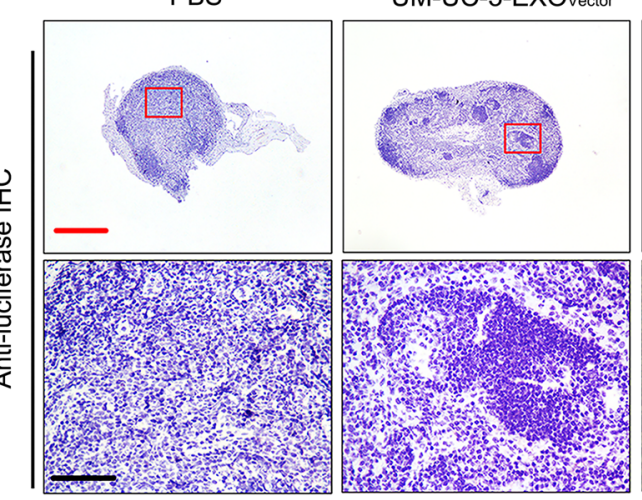

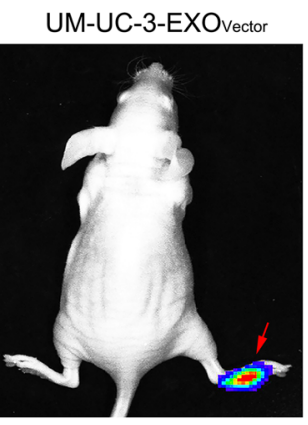
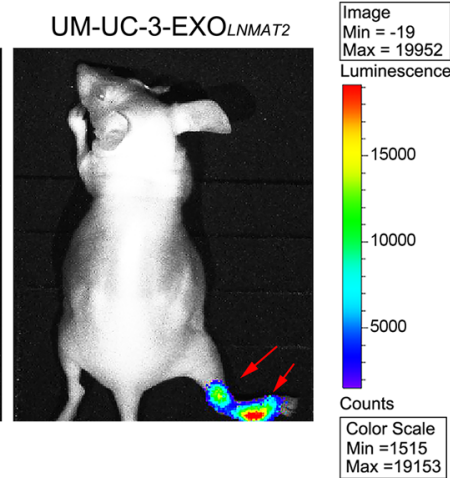

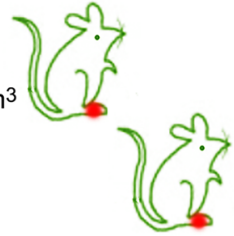

Enucleate

lymph nodes
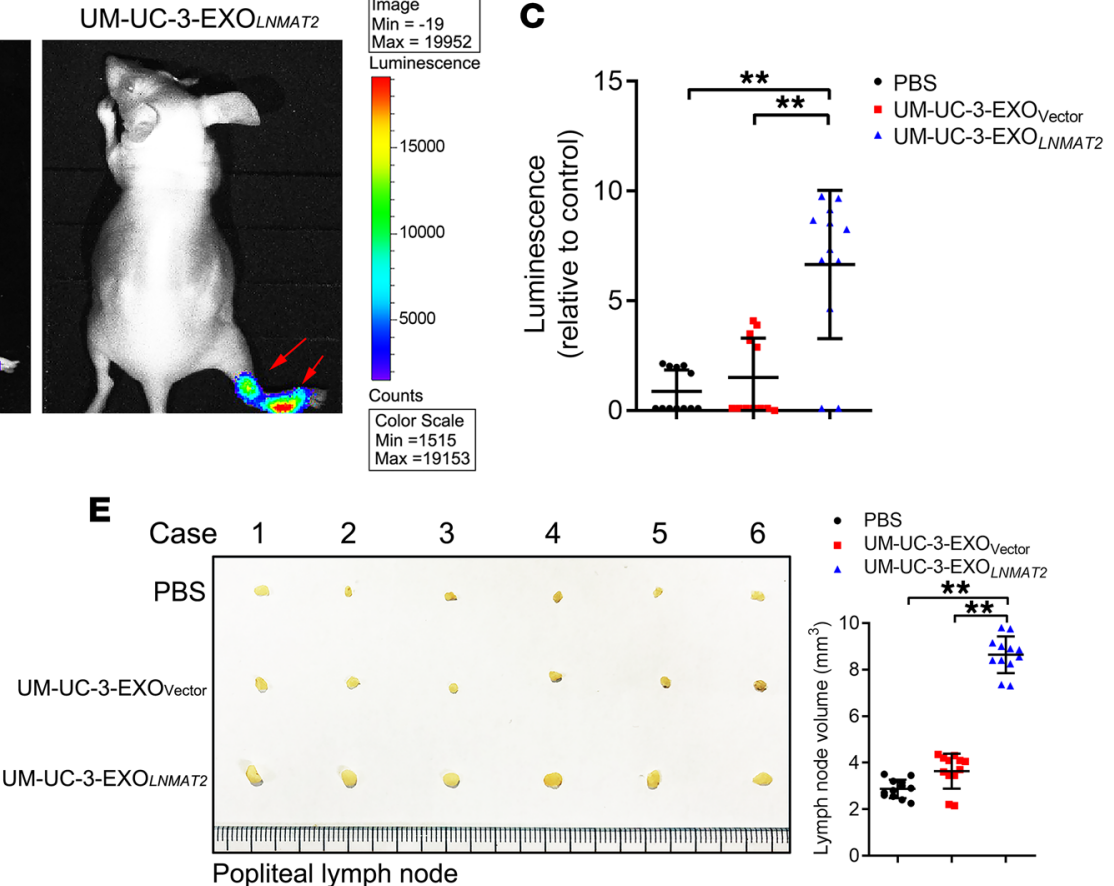

- PBS

- UM-UC-3-EXO

- UM-UC-3-EXO Lector

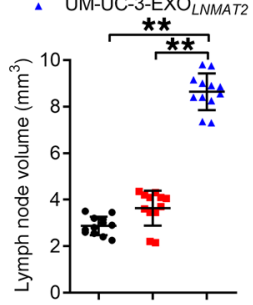

Popliteal lymph node

UM-UC-3-EXOLNMAT2

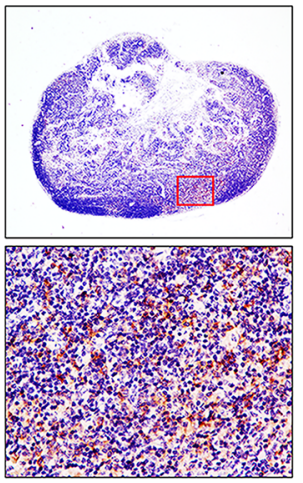

G

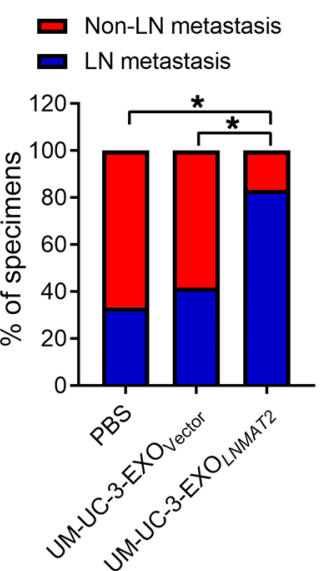

Figure 4. Exosomal LNMAT2 promotes lymphatic metastasis in vivo. (A) Schematic representation of establishment of the nude mouse model of popliteal LN metastasis. Representative bioluminescence images (B) and histogram analysis (C) of popliteal metastatic LN from nude mice treated with PBS, UM-UC-3-EXO ${ }_{\text {Vector }}$ or UM-UC-3-EXO ${ }_{\text {LNMAT2 }}$ after UM-UC-3 cells had been inoculated into the footpad $(n=12)$. Red arrow indicates footpad tumor and metastatic popliteal LN. Statistical significance was assessed using 1-way ANOVA followed by Dunnett's tests. (D) Representative image of the popliteal LN metastasis model. (E) Representative images of enucleated popliteal LNs (left) and histogram analysis (right) of the LN volume of all groups ( $n=12$ ). Statistical significance was assessed using 1-way ANOVA followed by Dunnett's tests. (F) Representative images of IHC staining with anti-luciferase antibody $(n=12)$. Scale bars: $500 \mu \mathrm{m}$ (red) or $50 \mu \mathrm{m}$ (black). (C) Percentages of LN status in all groups $(n=12)$. Statistical significance was assessed by $\chi^{2}$ test. Error bars represent the SD of 3 independent experiments. ${ }^{*} P<0.05 ;{ }^{* *} P<0.01$. 
A

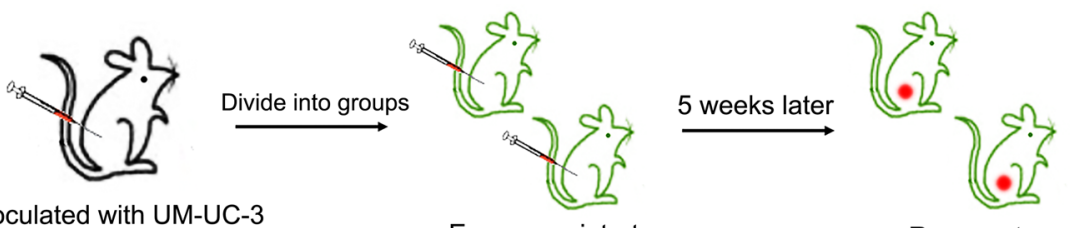

Inoculated with UM-UC-3

Exosomes intratumor

Remove tumors

subcutaneously

injection (Every 3 days)
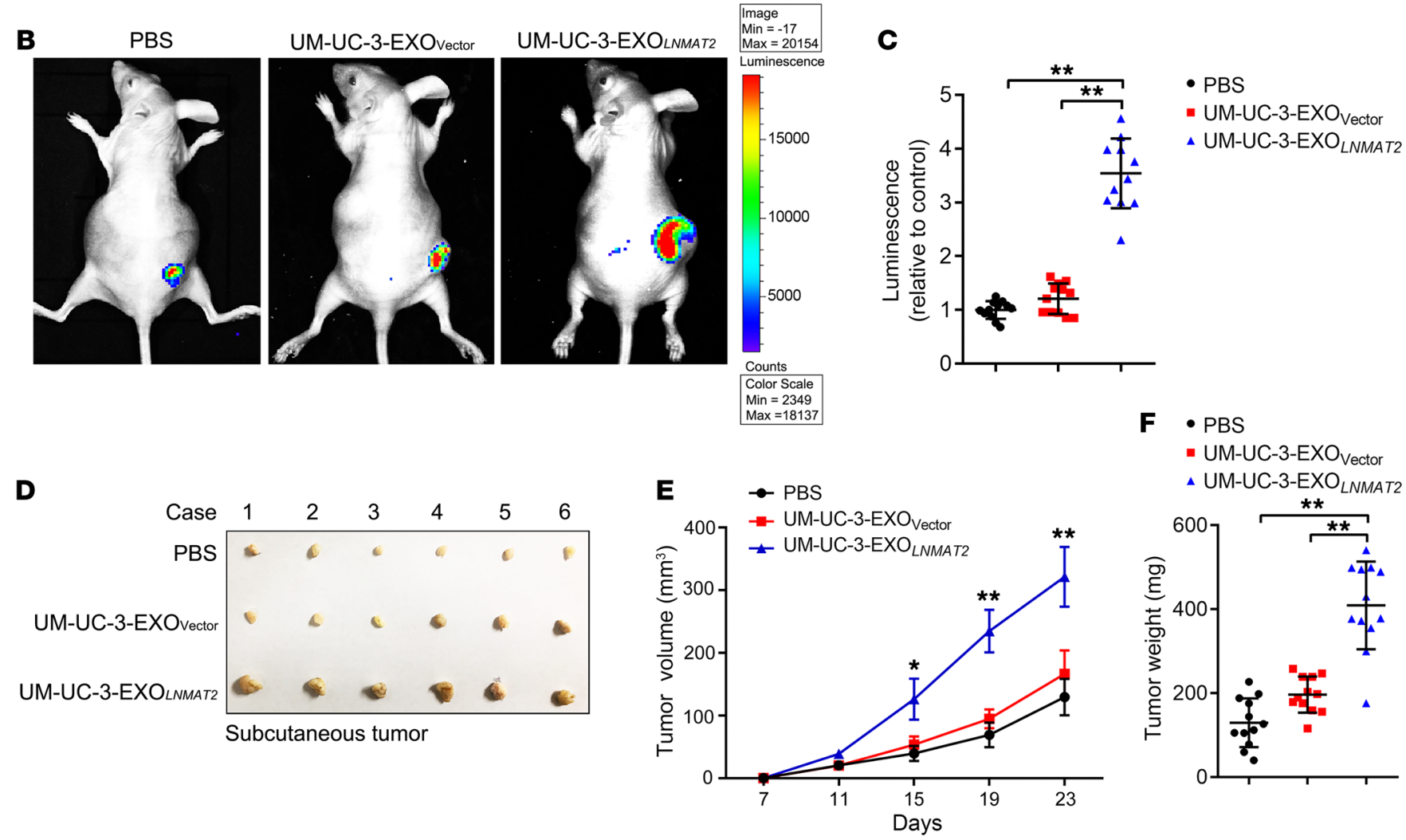

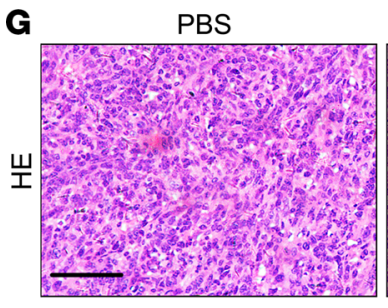

UM-UC-3-EXOvector
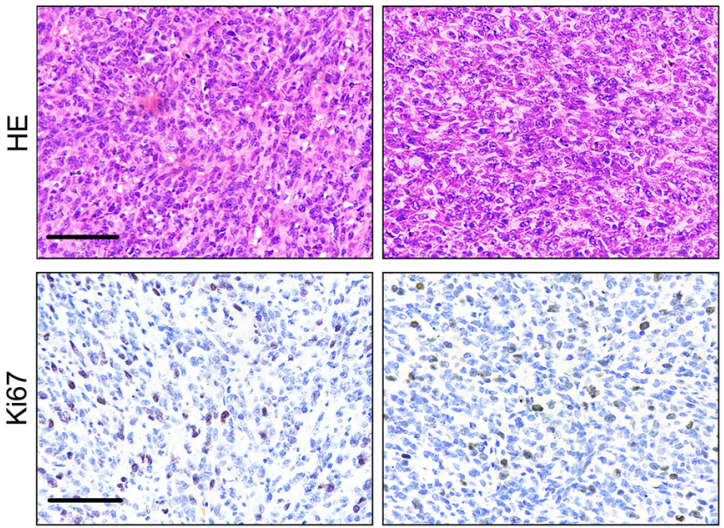

UM-UC-3-EXO NMAT2

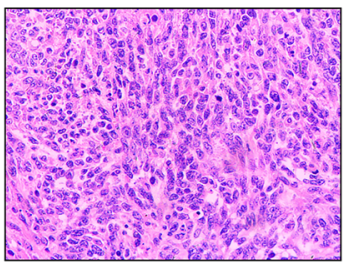

H $\quad$ PBS

- UM-UC-3-EXO Vector

$\triangle$ UM-UC-3-EXO ${ }_{\text {LNMAT2 }}$

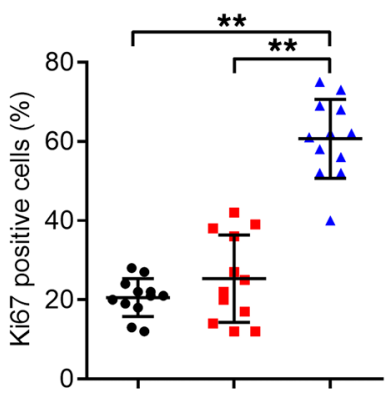

Figure 5. Exosomal LNMAT2 promotes BCa tumorigenesis in vivo. (A) Schematic representation of the establishment of the xenograft model. Representative bioluminescence images (B) and histogram analysis (C) of subcutaneous tumors from nude mice treated with PBS, UM-UC-3-EXO ${ }_{\text {vector }}$, or UM-UC-3$\operatorname{EXO}_{\text {LNMAT2 }}(n=12)$. Statistical significance was assessed using 1-way ANOVA followed by Dunnett's tests. (D) Representative images of gross appearance of subcutaneous tumors from nude mice treated with PBS, UM-UC-3-EXO ${ }_{\text {Vector' }}$ or UM-UC-3-EXO LNMAT2 $_{2}(n=12)$. Measured tumor volumes $(\mathbf{E})$ and weights $(\mathbf{F})$ $(n=12)$. Statistical significance was assessed using 1-way ANOVA followed by Dunnett's tests. Representative images (C) and histogram analysis (H) of IHC staining for Ki67 expression $(n=12)$. Scale bars: $50 \mu \mathrm{m}$. Statistical significance was assessed using 1-way ANOVA followed by Dunnett's tests. Error bars represent the SD of 3 independent experiments. ${ }^{*} P<0.05 ;{ }^{* *} P<0.01$. 
higher expression levels of the proliferation marker Ki67 as compared

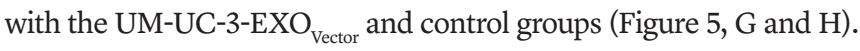
Collectively, these results indicate that BCa cell-secreted exosomal $L N M A T 2$ can promote lymphangiogenesis and $L N$ metastasis in vivo.

LNMAT2 interacted directly with hnRNPA2B1. Next, we investigated the molecular mechanism and interacting partners of LNMAT2 in BCa. In vitro RNA pull-down assays with biotinylated LNMAT2 and antisense control showed an obvious $35-40 \mathrm{kDa}$ band (Figure 6A), which mass spectrometry (MS) confirmed was hnRNPA2B1 (Figure 6B). Western blot analysis of LNMAT2-enriched proteins after RNA pull-down indicated that LNMAT2 bound specifically to hnRNPA2B1 (Figure 6, C and D). Consistently, confocal microscopy of LNMAT2 FISH and hnRNPA2B1 immunostaining showed that LNMAT2 and hnRNPA2B1 colocalized mostly in the cytoplasm of BCa cells (Figure 6E). RNA immunoprecipitation (RIP) showed enrichment of LNMAT2 by hnRNPA2B1, validating the interaction between LNMAT2 and hnRNPA2B1 (Figure 6F and Supplemental Figure 6A). Moreover, serial deletion analysis determined that the 1900-2100 nt region of LNMAT2 was indispensable for direct interaction with hnRNPA2B1 (Figure 6, G and $\mathrm{H}$ ). hnRNPA2B1, an RNA-binding protein (RBP), is involved in cytoplasmic RNA trafficking through the recognition of specific sequences on the target RNA (26). Sequence analysis by POSTAR2 $(27,28)$ indicated a sequence motif and structural preference of the RBP binding site for hnRNPA2B1 (Figure 6I), which was located in the 1930-1960 nt region of LNMAT2 and formed a stem-loop structure (Figure 6J). RIP performed after site-directed mutagenesis of this region revealed that it was critical to LNMAT 2 interaction with hnRNPA2B1 (Figure 6K and Supplemental Figure 6B).

hnRNPA2B1 mediated LNMAT2 packaging into exosomes. RNAs are selectively loaded into exosomes by $\operatorname{RBPs}(29,30)$, including hnRNPA2B1. Accordingly, we examined whether the direct interaction of $L N M A T 2$ with hnRNPA2B1 contributed to the packaging of LNMAT2 into exosomes. As shown in Figure 7A and Supplemental Figure 6C, hnRNPA2B1 knockdown reduced LNMAT2 levels only in the exosomes secreted by BCa cells but had no effect on LNMAT2 expression in BCa cells. hnRNPA2B1 sorts RNAs into exosomes by recognizing a specific motif (i.e., GGAG) (29), which is present on the hnRNPA2B1 binding sites (1930-1960 nt) of LNMAT2. Therefore, we induced mutation at these sites and observed significantly decreased $\mathrm{BCa}$ cell production of exosomal LNMAT2, indicating that the interaction between hnRNPA2B1 and these binding sites is important for LNMAT2 packaging into exosomes (Figure 7B and Supplemental Figure 6D). Additionally, in comparison with $m i R-18 a$, an miRNA retained in cells rather than loaded into exosomes (29), LNMAT2 presented a much higher exosome-to-cell ratio (Figure $7 \mathrm{C}$ and Supplemental Figure $6 \mathrm{E}$ ), which is similar to the report by Villarroya-Beltri et al., who demonstrated hnRNPA2B1-mediated exosome packaging of $m i R-198$ (29). Moreover, hnRNPA2B1 knockdown significantly decreased the enrichment of LNMAT2 and miR-198 in BCa cellsecreted exosomes (Figure 7D and Supplemental Figure 6F), indicating that LNMAT2 is specifically sorted into exosomes in an hnRNPA2B1-dependent manner.

Exosomal LNMAT2 was internalized by HLECs to induce lymphangiogenesis. As our results indicated that BCa cell-secreted exosomes promoted lymphangiogenesis, we then evaluated the inter- nalization of exosomes by HLECs. We labeled purified exosomes with PKH67 green fluorescent dye and incubated them with HLECs for 12 hours. Confocal images showed the green fluorescent punctate signal in the cytoplasm of recipient HLECs, indicating internalization of the PKH67-labeled exosomes, whereas no PKH67 fluorescent signal was observed in the control group, suggesting that the HLECs internalized the BCa cell-secreted exosomes (Figure 7E). We further examined whether exosomal LNMAT2 was successfully transferred into the HLECs and found that LNMAT2 expression was significantly increased in the HLECs after incubation with the exosomes (Figure 7F). Furthermore, LNMAT2 knockdown in exosomes secreted by 5637 cells diminished their ability to induce LNMAT2 overexpression (Figure 7G), whereas UM-UC-3-EXO ${ }_{L N M A T 2}$ increased LNMAT2 expression significantly in the HLECs (Supplemental Figure 7A).

To exclude the possibility that transcriptional activation of endogenous LNMAT2 in the HLECs activated the lymphangiogenesis, we established LNMAT2-KO cells from HLECs using the CRISPR-Cas9 approach with paired sgRNAs specifically targeting LNMAT2 (Supplemental Figure 7B). LNMAT2 deficiency was subsequently detected in the KO cells, suggesting successful inhibition of endogenous LNMAT2 expression (Figure $7 \mathrm{H}$ ). We then evaluated the effects of exosomal LNMAT2 on LNMAT2-WT and LNMAT2-KO HLECs. Tube formation and Transwell assays both demonstrated that, compared with exosomes secreted by si-NC-transfected 5637 cells $\left(5637-\mathrm{EXO}_{\text {si-NC }}\right.$ ), exosomes secreted by si-LNMAT2\#1-transfected 5637 cells (5637-EXO si-LNMAT2\#1 ) decreased LNMAT2-KO HLEC proliferation and migration (Figure 7, I-K), whereas UM-UC-3-EXO $\mathrm{ENMAT2}_{2}$ had a promoting effect on LNMAT2-KO HLECs as compared

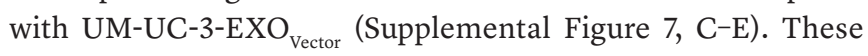
results were in accordance with those for the LNMAT2-WT HLECs (Figure 7, I-K and Supplemental Figure 7, C-E). Taken together, the results indicate that BCa cells induce lymphangiogenesis by transmitting exosomal LNMAT2 to HLECs.

Exosomal LNMAT2 upregulated PROX1 expression independently of VEGF-C. Previously, we demonstrated that VEGF-C produced by tumor cells (3) and TAMs (4) contributes to lymphangiogenesis and LN metastasis in BCa. However, more than $20 \%$ of BCa with LN metastasis presents with low VEGF-C expression (5). Our results showed that neither LNMAT2 overexpression nor LNMAT2 knockdown in both 5637 and UM-UC-3 cells induced VEGF-C mRNA or protein changes (Supplemental Figure 8, A-D), which suggested that exosomal LNMAT2-induced lymphangiogenesis and LN metastasis in BCa might be through a VEGF-Cindependent mechanism.

It has been proposed that PROX1 is essential for lymphatic vascular system development by regulating endothelial cell differentiation and metastatic dissemination (31, 32). Interestingly, qRT-PCR and Western blot analyses showed that PROX1 expression was significantly increased in exosomal LNMAT2-treated HLECs as compared with the control-treated HLECs (Figure 8, A and B, and Supplemental Figure 8, E and F), indicating that exosomal LNMAT2 upregulated PROX1 expression in HLECs.

Exosomal LNMAT2 formed a DNA-RNA triplex with the PROX1 promoter. To investigate the molecular mechanisms underlying exosomal LNMAT2-induced PROX1 expression in HLECs, we ana- 

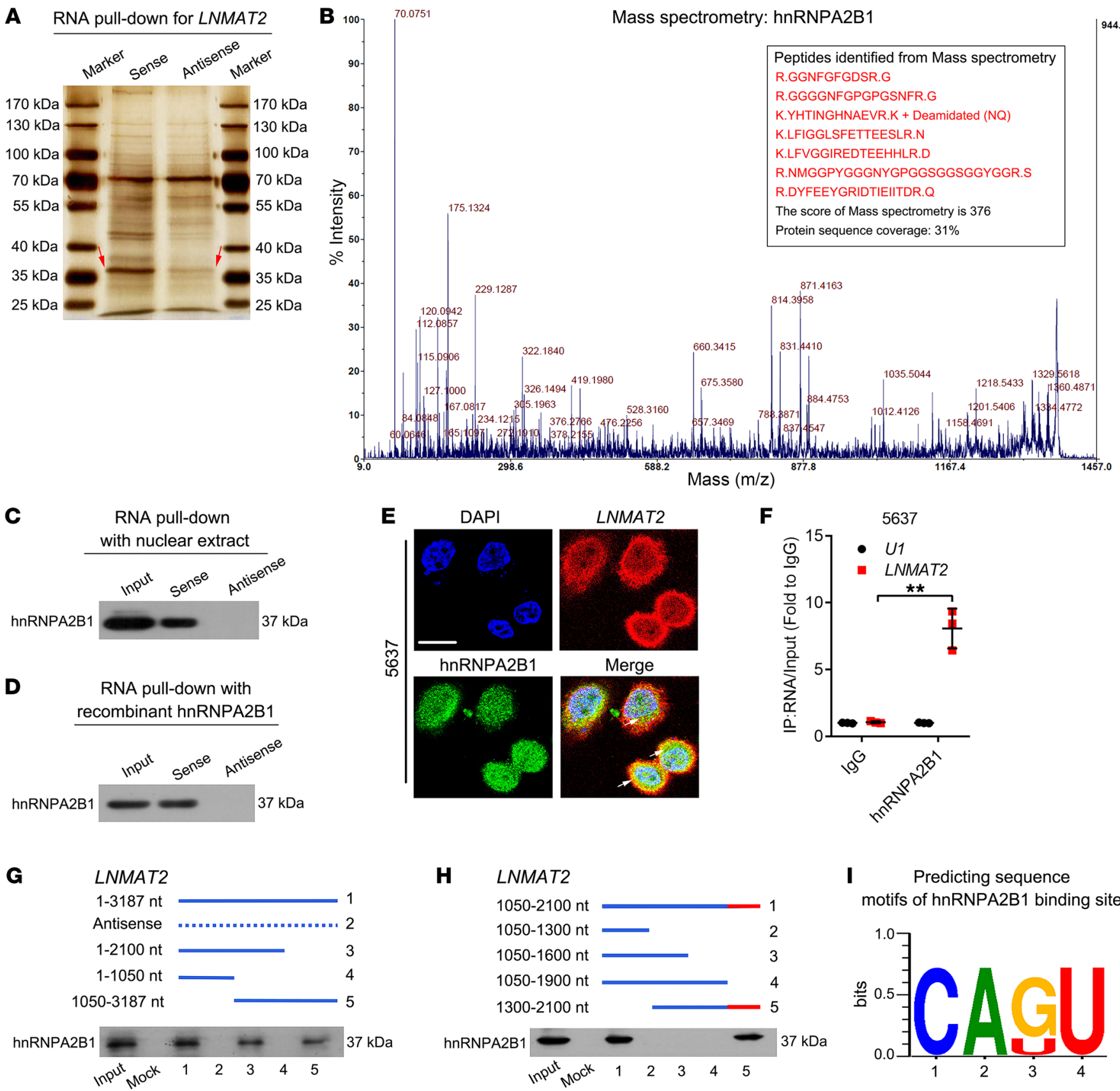

Predicting sequence
motifs of hnRNPA2B1 binding site
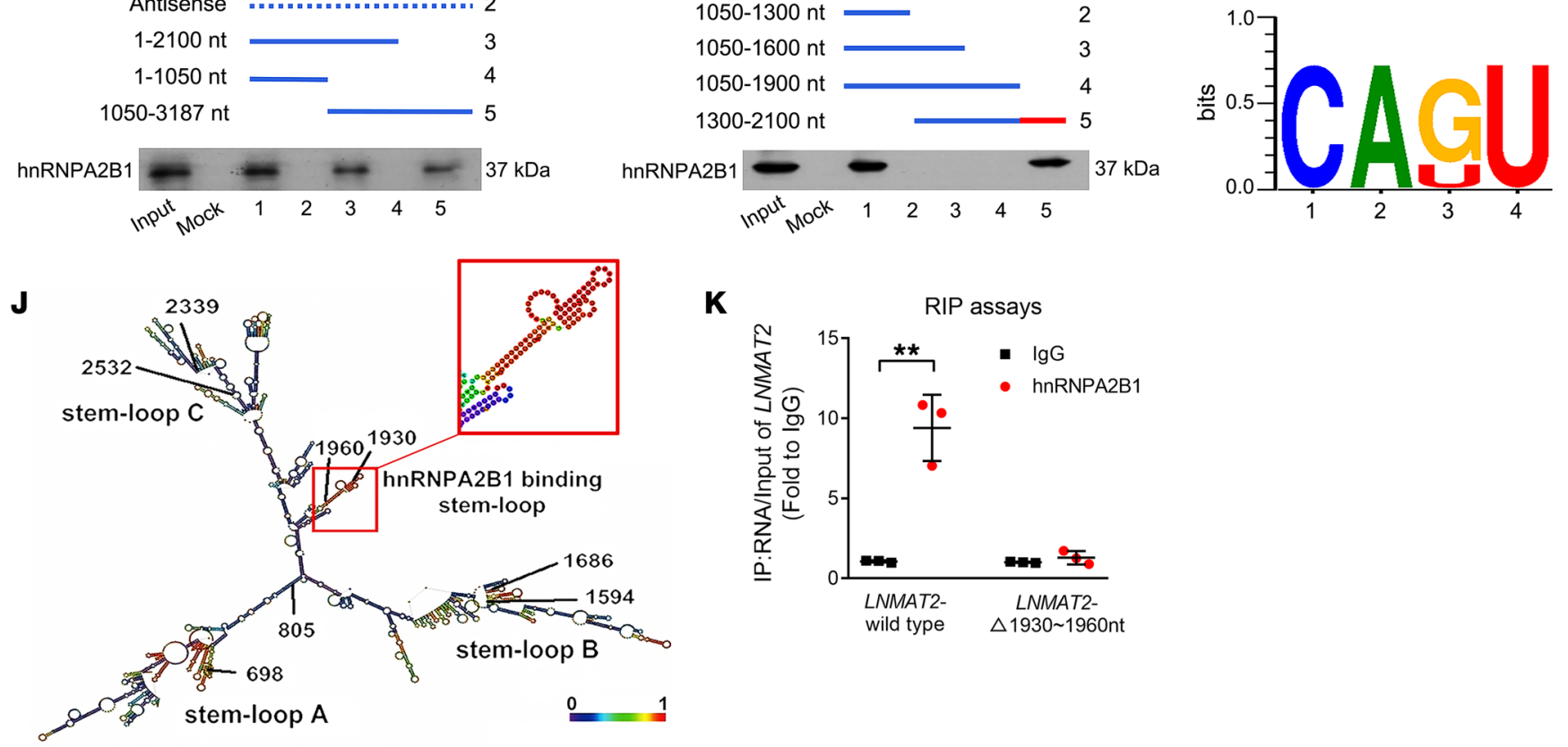
Figure 6. Direct interaction of LNMAT2 with hnRNPA2B1. (A) RNA pull-down assay using LNMAT2 sense and antisense RNAs in 5637 cells, followed by silver staining. Red arrows indicate hnRNPA2B1. (B) MS identification of LNMAT2-binding proteins. RNA pull-down and Western blot with 5637 cell nuclear extract (C) or purified recombinant hnRNPA2B1 (D) confirmed that $L N M A T 2$ was associated with hnRNPA2B1. (E) Fluorescence assessment of LNMAT2 and hnRNPA2B1 colocalization in 5637 cells. Scale bar: $5 \mu \mathrm{m}$. (F) RIP analysis using the anti-hnRNPA2B1 antibody revealing that $L N M A T 2$ interacted with hnRNPA2B1 in 5637 cells. Negative control, IgG; nonspecific control, U1. Statistical significance was assessed using 2-tailed Student's $t$ test. (G and $\mathbf{H}$ ) Serial deletions of LNMAT2 were used in RNA pull-down assays to identify regions required for $L N M A T 2$ and hnRNPA2B1 interaction. (I) POSTAR2 prediction of sequence motifs of hnRNPA2B1 binding sites. (J) RNAalifold predicted that LNMAT2 would have 4 stable stem-loop structures. The inset (framed in red) indicates the hnRNPA2B1 binding stem-loop structures in LNMAT2. (K) RIP assays performed after site-directed mutagenesis of 1930-1960 nt of LNMAT2 in 5637 cells. Statistical significance was assessed using 2-tailed Student's $t$ test. Error bars represent the SD of 3 independent experiments. ${ }^{*} P<0.05$; ${ }^{* *} P<0.01$.

lyzed the subcellular location of exosomal LNMAT2 after internalization by LNMAT2-KO HLECs. Abundant LNMAT2 was detected in the nucleus (Supplemental Figure 8, G and H), indicating that LNMAT2 released by $\mathrm{BCa}$ cell-secreted exosomes translocated into the HLEC nucleus and exerted essential functions. We generated a series of luciferase constructs containing various lengths of the PROX1 promoter sequences, which is located from $-2000 \mathrm{bp}$ upstream to +200 bp downstream of the transcriptional start site to explore whether exosomal LNMAT2 transcriptionally upregulated PROX1. Luciferase assays showed that the -650 bp to -350 bp region of PROX1 promoter led to an obvious increase of transcriptional activity in exosomal LNMAT2-induced HLECs (Figure 8C and Supplemental Figure 9A). Moreover, chromatin isolation by RNA purification (ChIRP) assays demonstrated that exosomal LNMAT2 interacted physiologically with the P3 (-607 bp to $-597 \mathrm{bp}$ ) region in the PROX1 promoter in HLECs (Figure 8, D-F).

To further investigate whether LNMAT2 directly interacted with PROX1 promoter, we obtained 5 potential pairs of triplex-forming oligonucleotides (TFOs) and their corresponding triplex target sites (TTSs) in the exosomal LNMAT2 and PROX1 promoter from LongTarget (33), a tool for predicting IncRNA-DNA binding motifs, and each binding motif was subjected to fluorescence resonance energy transfer (FRET) analysis and circular dichroism (CD) spectroscopy (Supplemental Table 5). As shown in Figure 8G and Supplemental Figure 9B, in comparison with the single-stranded RNA (ssRNA)/PROX1 NC group, the fluorescence signal was markedly increased at $570-580 \mathrm{~nm}$ and reduced at $520 \mathrm{~nm}$ in the LNMAT2 (+2058 to +2069 nt)/PROX1 TTS1 (-607 to $-597 \mathrm{bp)}$ group in FRET analysis. These results were in accordance with the FENDRR/PITX2 positive control group (Supplemental Figure $9 \mathrm{C}$ and ref. 34), indicating energy transfer from the fluorescein donor to the rhodamine acceptor, and supporting the formation of a triplex structure (4). CD spectroscopy demonstrated a strong positive peak at $270-280 \mathrm{~mm}$; a deep negative peak at $210 \mathrm{~nm}$ was recorded in the LNMAT2 (+2058 to +2069 nt)/PROX1 TTS1 (-607 to -597 bp) group (Figure 8H and Supplemental Figure 9D), which was in accordance with the FENDRR/PITX2 positive control group (Supplemental Figure 9E). This validated the premise that the triplex was constructed between LNMAT2 and the PROX1 promoter sequences in vitro. Additionally, exosomal LNMAT2 enhanced the luciferase intensity of the PROX1 promoter, while no obvious change was observed in the mutated PROX1 promoter (PROX1-P3), suggesting that the sequence between -607 and -597 bp in the PROX1 promoter is of great significance for exosomal LNMAT2-induced PROX1 transactivation (Figure 8, I and J, and Supplemental Figure 9, F and G). Together, these data suggest that exosomal LNMAT2 can form a triplex with the lymphatic $P R O X 1$ promoter sequence and upregulate its transcription levels.

Exosomal LNMAT2 interacted with hNRNPA2B1 to promote H3K4 trimethylation at the PROX1 promoter. hnRNPA2B1 is involved in H3K4 trimethylation-associated (H3K4me3-associated) epigenetic regulation by binding to the target DNA $(35,36)$. Accordingly, we explored whether hnRNPA2B1 contributes to exosomal LNMAT2-induced transactivation of PROX1 by regulating H3K4me3 levels in the PROX1 promoter region in HLECs. ChIP indicated enrichment of the $P R O X 1$ promoter sequences associated with hnRNPA2B1 and H3K4me3 in HLECs treated with exosomes secreted by LNMAT2-transfected 5637 cells $\left(5637-\mathrm{EXO}_{\text {LNMAT2 }}\right)$ and

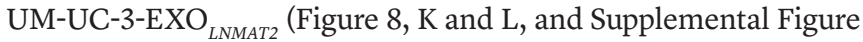
9, $\mathrm{H}$ and I), whereas there was a significant reduction in PROX1 promoter sequences associated with hnRNPA2B1 and H3K4me3

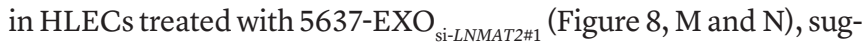
gesting that exosomal LNMAT2 upregulates PROX1 expression by interacting directly with hnRNPA2B1 to increase H3K4me3 levels in the PROX1 promoter.

Exosomal LNMAT2-induced PROX1 upregulation promoted lymphatic metastasis. Next, we clarified whether PROX1 is required for exosomal LNMAT2-induced lymphangiogenesis in BCa. Reducing LNMAT2 expression in exosomes diminished tumor-induced HLEC proliferation and migration, and ectopic PROX1 rescued the effects (Figure 9, A-C). In contrast, PROX1 silencing abolished exosomal LNMAT2-induced HLEC tube formation and migration significantly and independently of VEGF-C (Supplemental Figure 10, A and B). These results suggested that PROX1 was required for exosomal LNMAT2-mediated VEGF-C-independent lymphangiogenesis and lymphatic metastasis in vitro. Furthermore, in vivo assays showed that the UM-UC-3-EXO ${ }_{L N M A T 2}$ group exhibited larger-volume popliteal LNs relative to the UM-UC-3-EXO $\mathrm{Evector}_{\text {- }}$ group after both had been treated with VEGF-C-neutralizing antibody (pV1006R-r) (Figure 9, D-F). IHC of mouse BCa tissues also demonstrated that high LNMAT2 levels were accompanied by increased PROX1 expression in LYVE-1-positive lymphatic vessels in both the intratumoral and peritumoral regions (Figure 9, G-I). The UM-UC-3-EXO ${ }_{L N M A T 2}$ group showed more enhancing effects on LN metastasis relative to the UM-UC-3-EXO $\mathrm{Evector}_{\text {group after }}$ pV1006R-r treatment in both, resulting in shorter survival times (Figure 9, J and K, and Supplemental Table 6). These results provide further evidence that exosomal LNMAT2 upregulates PROX1 expression to induce BCa lymphatic metastasis in a VEGF-Cindependent manner.

Exosomal LNMAT2 was associated with LN metastasis in BCa patients. Accumulating evidence shows that BCa cell-secreted exosomal IncRNAs can be detected in urine, and these urinary exosomal lncRNAs have been recognized as promising biomarkers for early diagnosis of $\mathrm{BCa}$ (37). Therefore, clarifying the clinical 
A

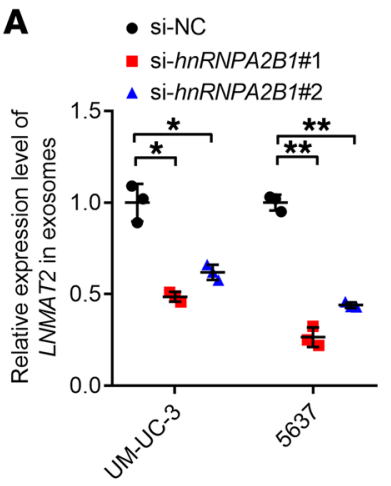

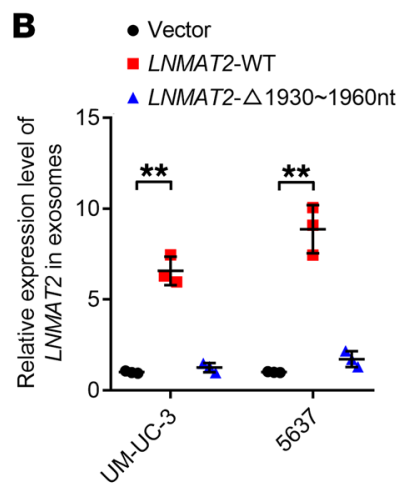

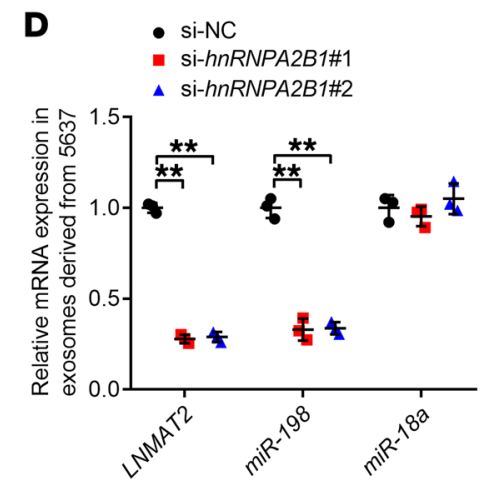

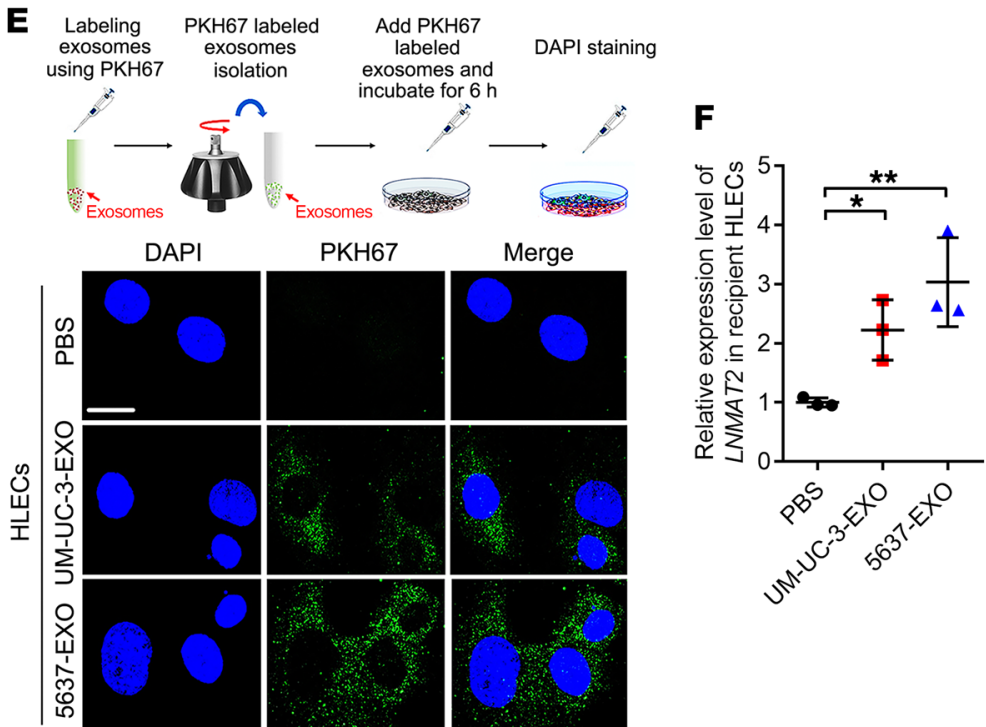

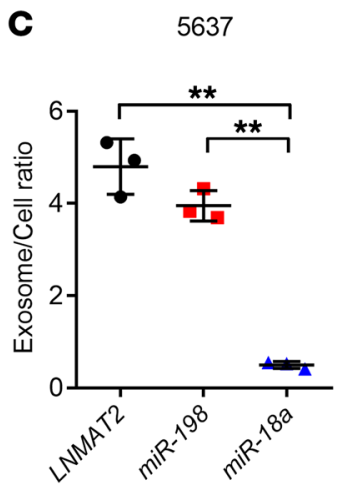

G

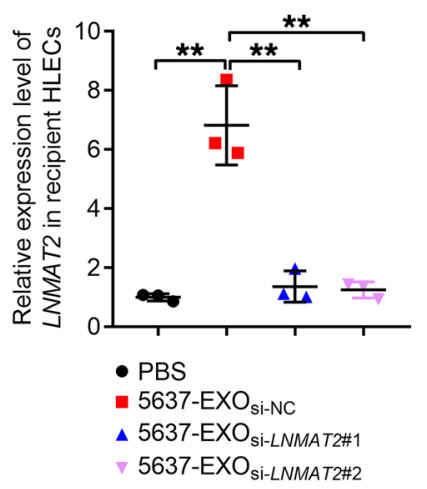

H

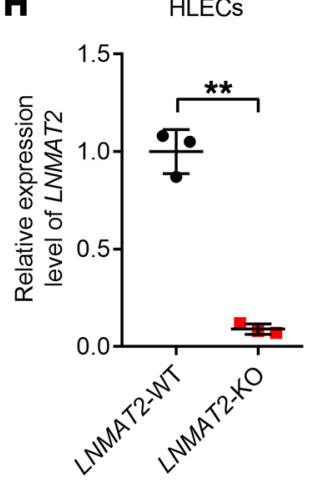

I

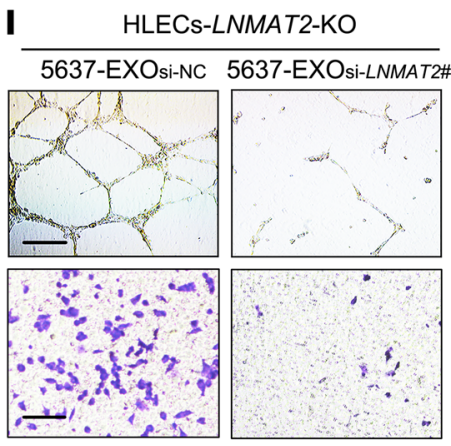

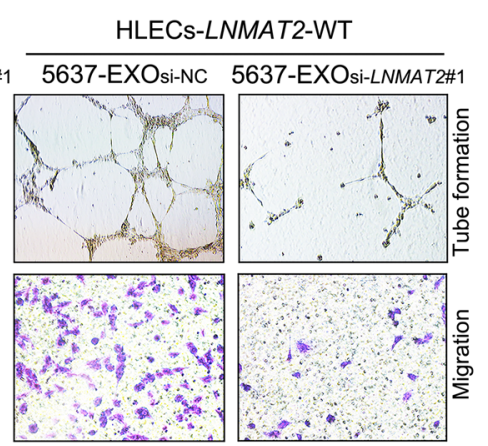

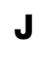

$\mathbf{J}$

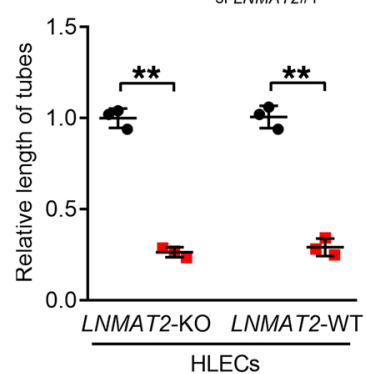

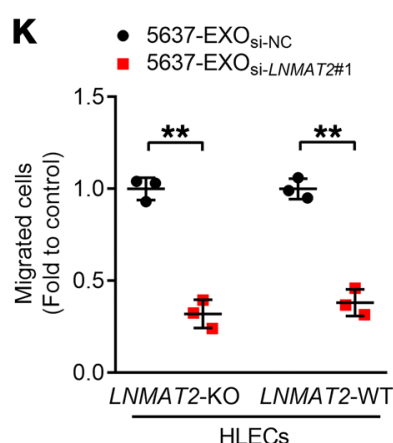

Figure 7. LNMAT2 is packaged into exosomes in an hnRNPA2B1-dependent manner and transported to HLECs. (A) qRT-PCR analysis of LNMAT2 expression in exosomes secreted by hnRNPA2B1 knockdown cells. Statistical significance was assessed using 1-way ANOVA followed by Dunnett's tests. (B) qRTPCR analysis of LNMAT2 expression in BCa cell-secreted exosomes. Statistical significance was assessed using 1-way ANOVA followed by Dunnett's tests. (C) The exosome/cell ratio of RNAs in 5637 cells obtained by qRT-PCR. Statistical significance was assessed using 1-way ANOVA followed by Dunnett's tests. (D) qRT-PCR analysis of RNA levels in exosomes secreted by hnRNPA2B1 knockdown 5637 cells. Statistical significance was assessed using 1-way ANOVA followed by Dunnett's tests. (E) Schematic illustration of exosome internalization assays and representative images of HLEC fluorescence after incubation with PKH67-labeled (green) BCa cell exosomes. Scale bar: $5 \mu \mathrm{m}$. qRT-PCR analysis of LNMAT2 expression in HLECs treated with PBS, 5637-EXO,

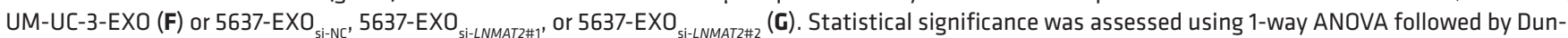
nett's tests for multiple comparisons. (H) qRT-PCR confirming the LNMAT2 knockout. Statistical significance was assessed using 2-tailed Student's $t$ test. Representative images (I) and quantification of tube formation (J) and Transwell migration (K) by HLECs (LNMAT2-KO or LNMAT2-WT) after treating with

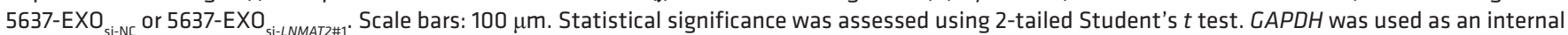
control for qRT-PCR analysis in A-H. Error bars represent the SD of 3 independent experiments. ${ }^{*} P<0.05$; ${ }^{* *} P<0.01$. 


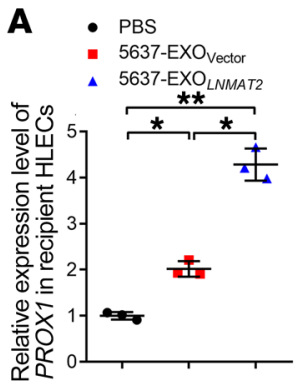

B

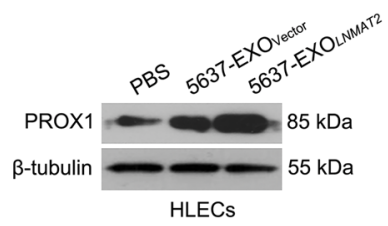

C
Luciferase assays in HLECs

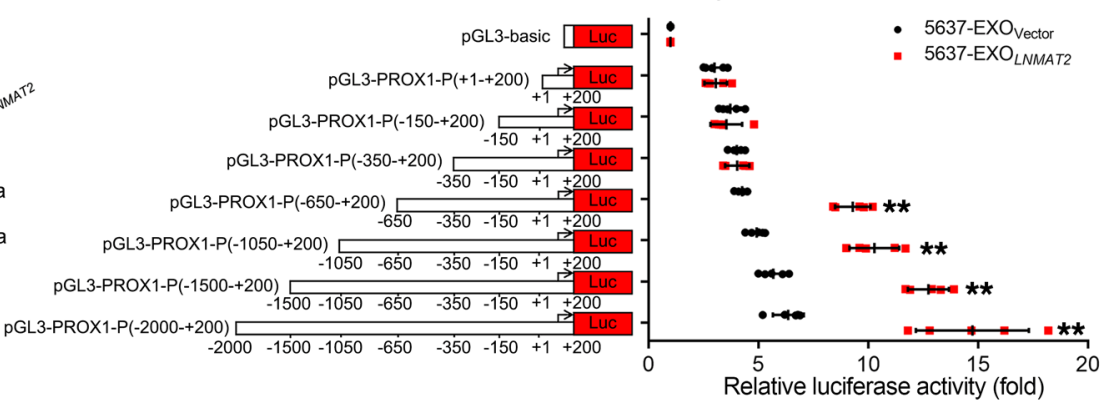

D

E

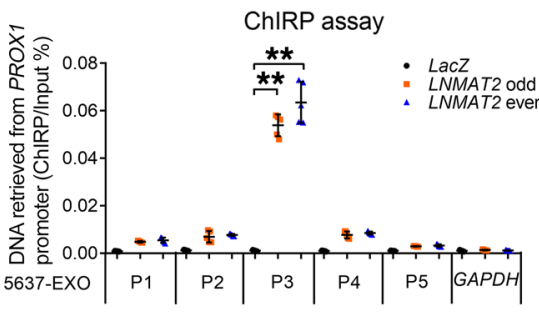

$\mathbf{F}$

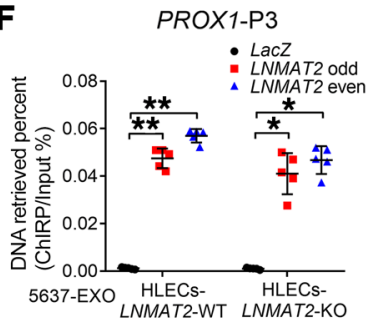

G

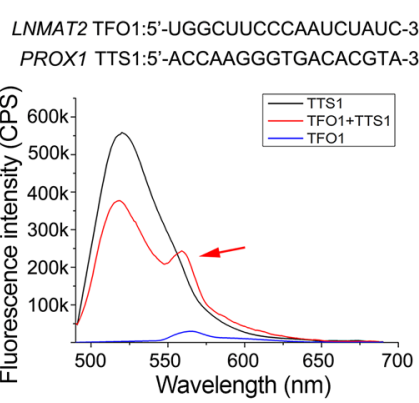

$\mathbf{K}$

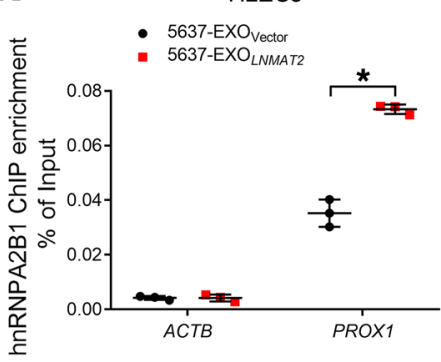

H

LNMAT2 TFO1:5'-UGGCUUCCCAAUCUAUC-3' PROX1 TTS1:5'-ACCAAGGGTGACACGTA-3'

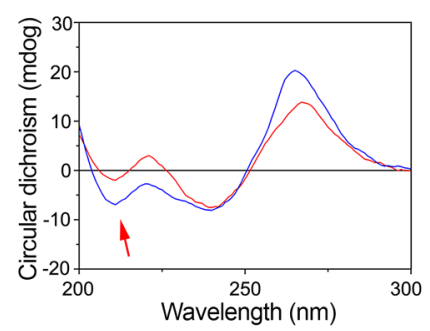

$\mathbf{L}$

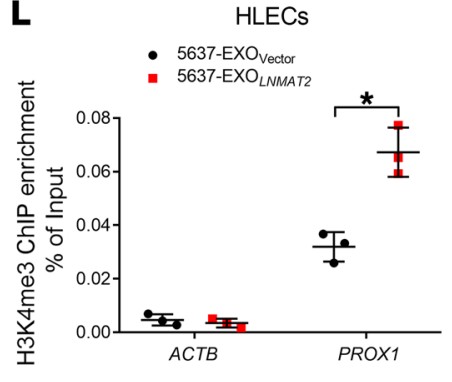

I

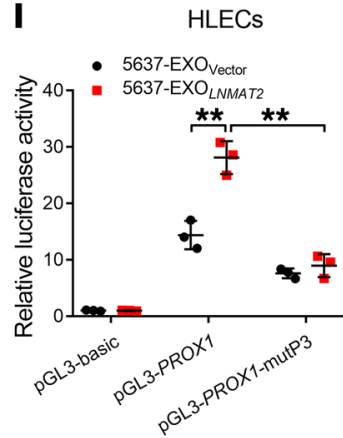

M

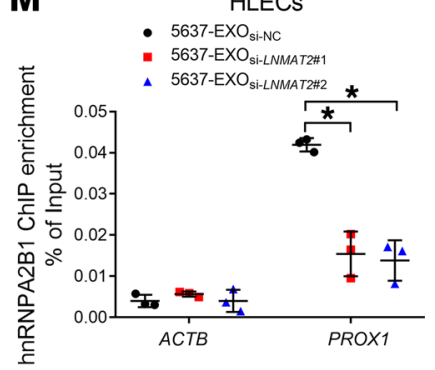

J
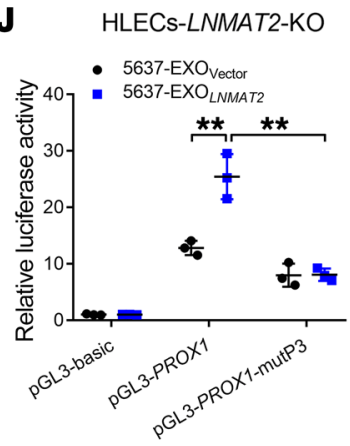

$\mathbf{N}$

HLECS

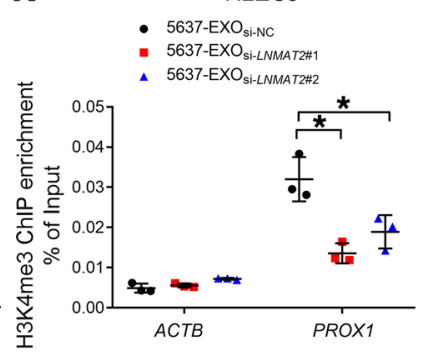

Figure 8. Exosomal LNMAT2 forms a DNA-RNA triplex with the PROX1 promoter. qRT-PCR (A) and Western blot (B) of PROX1 expression in HLECs treated with PBS, 5637-EXO ${ }_{\text {Vector' }}$ or 5637-EXO LNMAT2. GAPDH was used as an internal control in qRT-PCR. Statistical significance was assessed using 1-way ANOVA followed by Dunnett's tests. (C) Sequential deletions for evaluating the transcriptional activity of the $P R O X 1$ promoter in HLECs treated with 5637-EXO ${ }_{\text {vector }}$ or

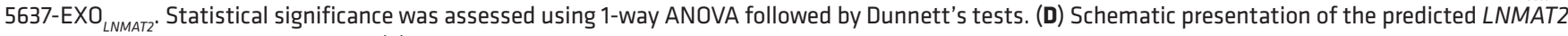
binding sites in the PROX1 promoter. (E) ChIRP of LNMAT2-associated chromatin in HLECs treated with 5637-EXO. Statistical significance was assessed using 2-tailed Student's $t$ test. (F) ChIRP of LNMAT2-associated chromatin in LNMAT2-WT or LNMAT2-KO HLECs treated with 5637-EXO. Statistical significance was assessed using 2-tailed Student's $t$ test. (C) FRET of a 1:5 mixture (red) of TFO (black) in LNMAT2 with TTS (blue) in the PROX1 promoter. (H) CD spectrum of a 1:1 mixture of TFO in LNMAT2 with TTS in the PROX1 promoter (red). The sum of the TFO and TTS is shown in blue. Evaluation of WT

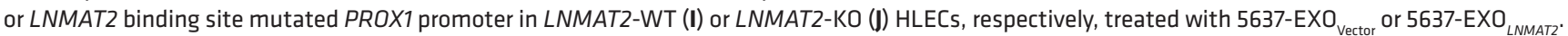
Statistical significance was assessed using 1-way ANOVA followed by Dunnett's tests. ChIP-qPCR of hnRNPA2B1 occupancy (K and M) and H3K4me3 (L and N) status in the PROX1 promoter after HLEC incubation with indicated exosomes. Statistical significance was assessed using 2-tailed Student's $t$ test and 1-way ANOVA followed by Dunnett's tests for multiple comparisons. Error bars represent the SD of 3 independent experiments. ${ }^{*} P<0.05 ;{ }^{* *} P<0.01$.

significance of exosomal LNMAT2 in BCa LN metastasis would be of great significance. First, we found that LNMAT2 overexpression was associated with increased lymphatic vessel density in both the intratumoral and peritumoral regions of BCa tissues in anoth- er 206-case cohort (Figure 10, A and B). Interestingly, LNMAT2 expression in urinary-EXO correlated positively with that in paired tumor tissues (Supplemental Figure 11A). Consistently, ISH showed significantly upregulated LNMAT2 expression in tumor tis- 
A
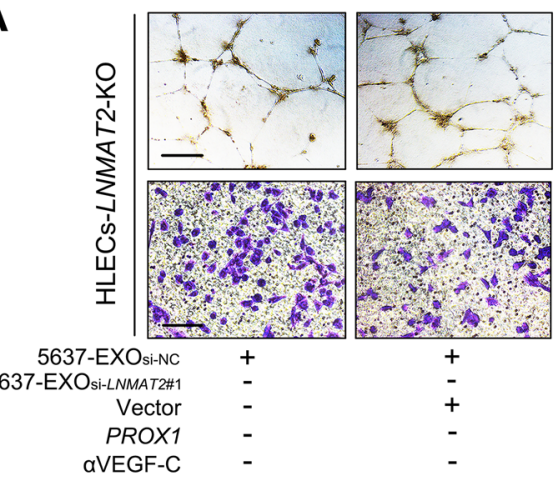

B

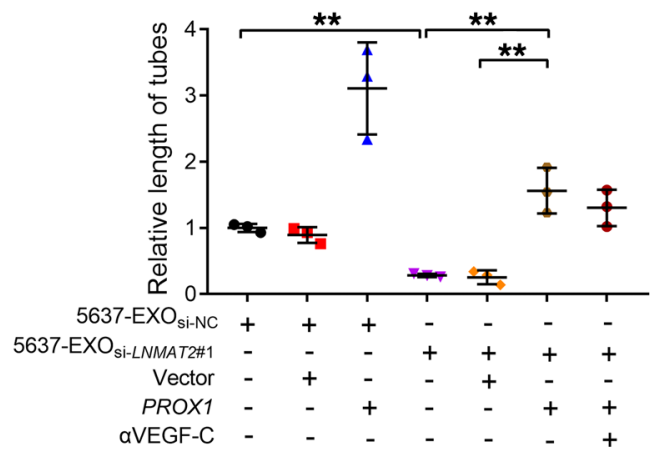

D

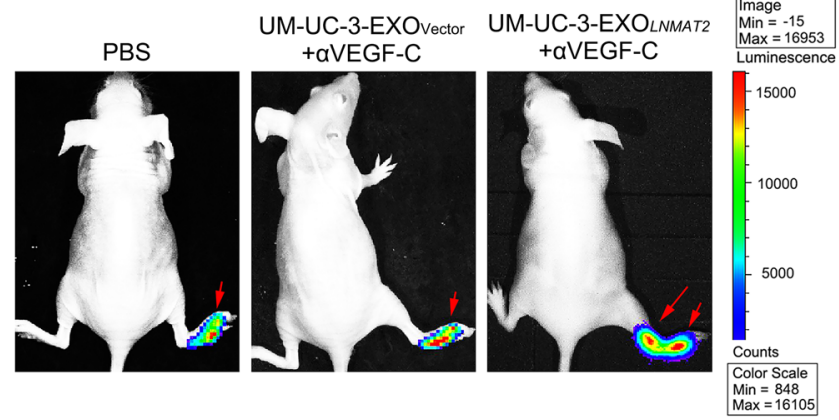

G

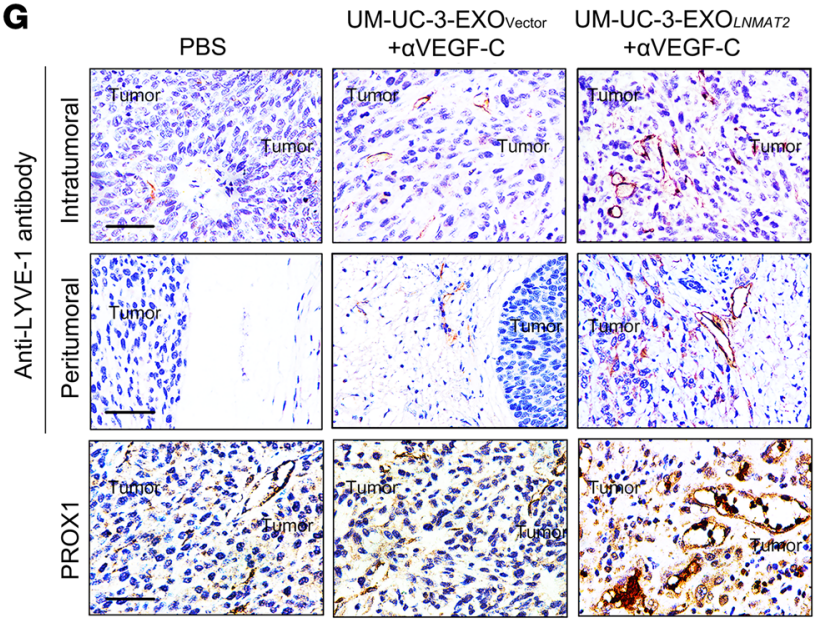

K

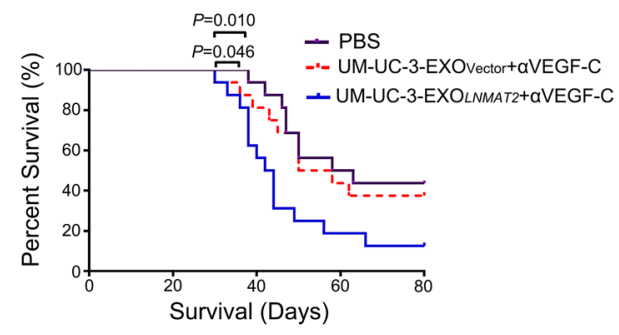

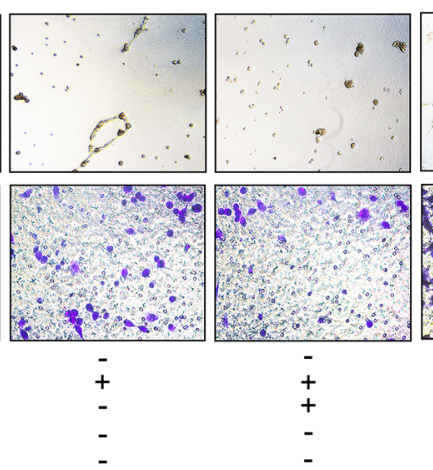

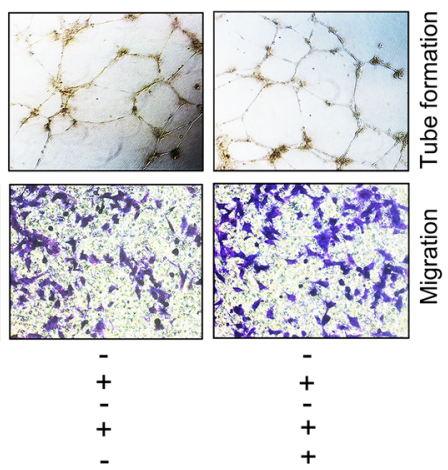

C

HLECS-LNMAT2-KO
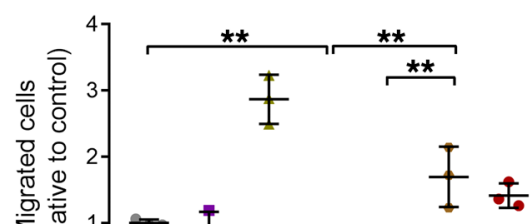

5637-EXO

$\begin{array}{rlllllll}\text { si-LNMAT2\#1 } & - & - & - & + & + & + & + \\ \text { Vector } & - & + & - & - & + & - & - \\ \text { PROX1 } & - & - & + & - & - & + & + \\ \text { aVEGF-C } & - & - & - & - & - & - & +\end{array}$

E PBS

F

- UM-UC-3-EXO Vector $+\alpha$ VEGF-C

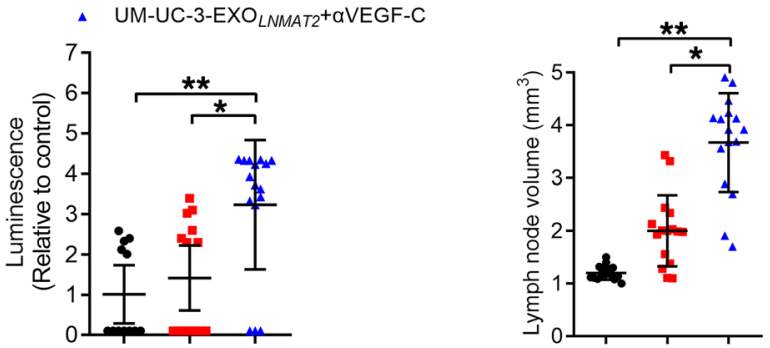

H
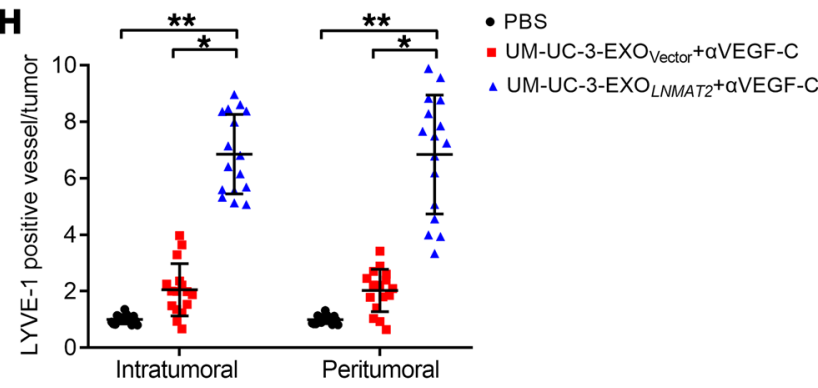

I L Low PROX1

J $\square$ Non-LN metastasis

口 High PROX1

bN metastasis
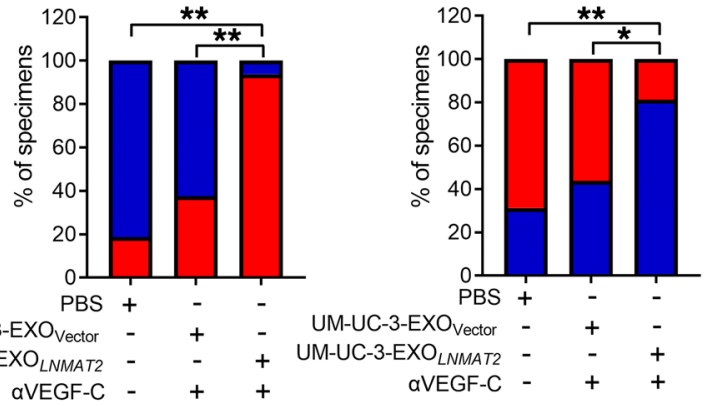
Figure 9. PROX1 is required for exosomal LNMAT2-mediated lymphangiogenesis. Representative images (A) and histogram analysis of tube formation (B) and Transwell migration (C) by LNMAT2-KO HLECs treated with 5637-EXO si-Nc or 5637-EXO si-LNMAT2\#1, $_{1}$ transfected with vector or PROX1 plasmid, or in which VEGF-C was inhibited. Scale bars: $100 \mu \mathrm{m}$. Statistical significance was assessed using 1-way ANOVA followed by Dunnett's tests. Representative bioluminescence images (D) and histogram analysis (E) of popliteal metastatic LN from nude mice treated with PBS, UM-UC-3-EXO ${ }_{\text {Vector }}$, or UM-UC-3-EXO ${ }_{\text {LNMAT2 }}$ with or without coinjection of VEGF-C neutralizing antibody after UM-UC-3 cells had been inoculated into the footpad $(n=16)$. Red arrow indicates footpad tumor and metastatic popliteal LN. Statistical significance was assessed using 1-way ANOVA followed by Dunnett's tests. (F) Histogram analysis of the LN volume ( $n$ = 16). Statistical significance was assessed using 1-way ANOVA followed by Dunnett's tests. Representative images (G) and histogram analysis of IHC staining evaluating lymphatic vessel density based on LYVE-1 $(\mathbf{H})$ and PROX1 expression (I) in footpad tumors $(n=16)$. Scale bars: $50 \mu \mathrm{m}$. Statistical significance was assessed using 1-way ANOVA followed by Dunnett's tests and the $\chi^{2}$ test. (J) The percentages of LN status in all groups $(n=16)$. Statistical significance was assessed by $\chi^{2}$ test. (K) Kaplan-Meier survival curve for control, UM-UC-3-EXO ${ }_{\text {Vector }}$ or UM-UC-3-EXO ${ }_{\text {LNMAT2 }}$ groups with or without inhibition of VEGF-C $(n=16)$. Error bars represent the SD of 3 independent experiments. ${ }^{*} P<0.05 ;{ }^{*} P<0.01$.

sues from BCa patients with high exosomal LNMAT2 as compared with patients with low exosomal LNMAT2 (Supplemental Figure 11, B and C), implying that exosomal LNMAT2 played a crucial role in LNMAT2-related LN metastasis in BCa. We explored whether exosomal LNMAT2 was clinically relevant to LN metastasis in BCa. Exosomal LNMAT2 expression in BCa patients with LN metastasis was dramatically higher than that in patients without LN metastasis (Figure 10C). Statistical analysis revealed a positive correlation between exosomal LNMAT2 and LN metastasis (Supplemental Table 7). Moreover, Kaplan-Meier analysis showed that high exosomal LNMAT2 level was associated with poor prognosis of $\mathrm{BCa}$ (Figure 10, D and E). Univariate and multivariate analysis also supported exosomal LNMAT2 as an independent prognostic factor of OS and DFS in BCa (Supplemental Tables 8 and 9). Receiver operating characteristic (ROC) analysis showed that urinary exosomal LNMAT2 could discriminate between patients with $\mathrm{BCa}$ and the healthy controls, and there was higher diagnostic accuracy, as measured by the AUC, for diagnosing LN metastasis in BCa (Figure 10, $\mathrm{F}$ and $\mathrm{G})$. Consistently, we also found that exosomal LNMAT2 was significantly elevated in BCa serum samples, and higher LNMAT2 expression was detected in serum exosomes (serum-EXO) from BCa patients with LN metastasis compared with that from patients without LN metastasis (Supplemental Figure 11, D and E). Importantly, serum exosomal LNMAT2 overexpression correlated with shorter OS in BCa patients (Supplemental Figure 11F). Taken together, our results suggest that exosomal LNMAT2 might be a potential diagnostic biomarker and therapeutic target for LN metastatic BCa (Figure 10H).

\section{Discussion}

Lymphangiogenesis, the growth of new lymphatic vessels, plays a crucial part in LN metastasis (38). Although tumor-associated lymphangiogenesis is driven mainly by VEGF-C, nearly $20 \%$ of BCa with LN metastasis show low VEGF-C expression $(5,6)$. So far, the mechanism of lymphatic metastasis of BCa cells with low
VEGF-C expression remains unknown. Herein, we demonstrated that a tumor-secreted exosomal lncRNA is involved in lymphangiogenesis and LN metastasis of BCa in a VEGF-C-independent manner. We identified a novel lncRNA, LNMAT2, which is enriched in urinary exosomes and plays an important role in $\mathrm{BCa}$ lymphatic metastasis. LNMAT2 was packaged into exosomes via direct interaction with hnRNPA2B1. Subsequently, exosomal LNMAT2 was internalized by HLECs and epigenetically activated PROX1 expression by recruiting hnRNPA2B1 to the PROX1 promoter, resulting in the lymphangiogenesis and lymphatic metastasis of BCa. These findings provide in-depth mechanistic and translational insights into the pathway by which exosomal lncRNA promotes $\mathrm{BCa}$ lymphatic metastasis in a VEGF-C-independent manner, and support the emergence of LNMAT2 as a novel therapeutic target in $\mathrm{BCa}$.

Exosomes have been extensively studied for their function in intercellular communication between the tumor and the tumor microenvironment (TME) (12). Previously, several independent studies have suggested that exosomal lncRNAs were involved in proliferation (39), chemoresistance (40), and stemness (41) in various cancers. Herein, we found that LNMAT2 was overexpressed in urinary-EXO and serum-EXO from patients with $\mathrm{BCa}$, and both urinary and serum exosomal LNMAT2 were positively associated with lymphatic metastasis in patients with $\mathrm{BCa}$, which indicated that exosomal RNA analysis could be utilized for early detection of LN metastasis. Our results showed that exosomal LNMAT2 promoted lymphangiogenesis and $\mathrm{LN}$ metastasis of $\mathrm{BCa}$, suggesting that LNMAT2 may represent a potential molecular target for clinical intervention in patients with $\mathrm{BCa}$ with $\mathrm{LN}$ metastasis.

It is well-established that LN metastasis is a major cause of BCa-related mortality, and intervention of LN metastasis might be a promising therapeutic strategy for improving the prognosis of BCa $(42,43)$. Herein, a tumor-secreted exosomal LNMAT2 emerged as a potent target for diminishing lymphangiogenesis and LN metastasis in $\mathrm{BCa}$, highlighting its attractive role in cancer treatment. We demonstrated that downregulating exosomal LNMAT2 expression in BCa cells via RNA interference inhibited tumor-associated lymphangiogenesis and might be a potential approach for suppressing LN metastasis. However, the stabilization and delivery efficiency of siRNAs in vivo is one of the most critical issues in oligonucleotide therapeutics. Locked nucleic acid (LNA) modification improved siRNA stability and pharmacokinetics, and could be exploited to facilitate siRNA delivery into target cells, resulting in effective suppression of tumor growth with minimal adverse effects in experimental animal models (44). Therefore, inhibiting the function of exosomal LNMAT2 via LNA-modified siRNA might develop a new strategy for treating LN metastasis in human cancer.

Exosomes regulate the biological functions of recipient cells via RNA transfer. The exosomal RNAs are selectively sorted into exosomes by several RBPs $(29,30)$, including hnRNPA2B1, which participates in exosomal RNA packaging by interacting with its target RNAs (29). It has been demonstrated that hnRNPA2B1 regulates the localization of miRNA into exosomes by binding to specific motifs (i.e., GGAG) (29). hnRNPA2B1 also plays a role in loading a lncRNA ( $\operatorname{lncARSR}$ ) into exosomes (45). In the present study, we proposed a model wherein hnRNPA2B1 binds specif- 
A
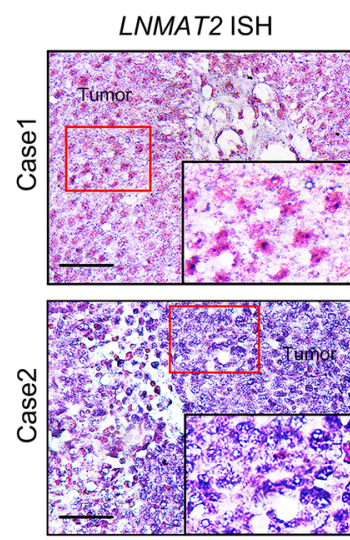

C

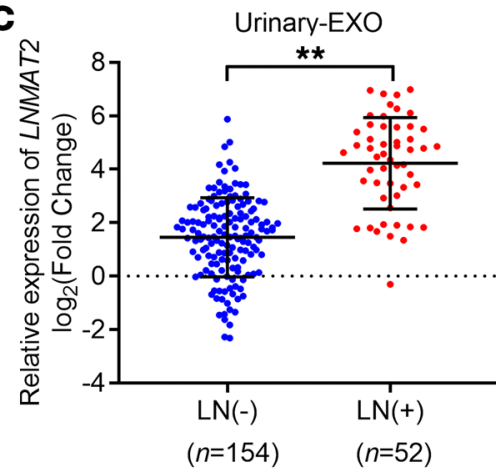

$\mathbf{F}$

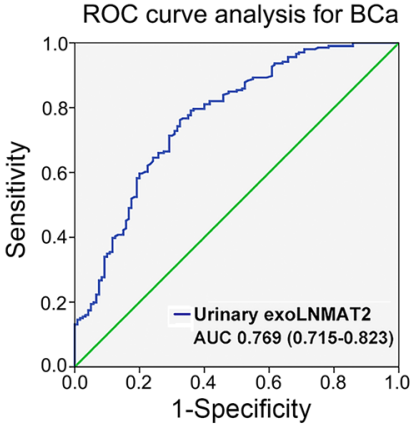

G

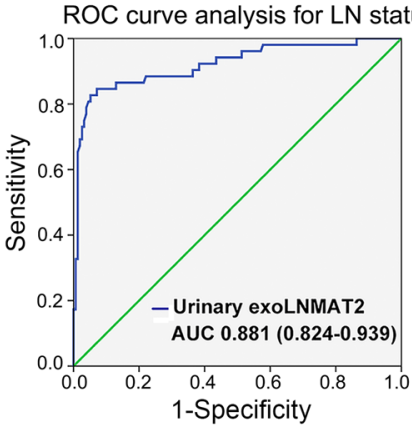

LYVE-1 IHC
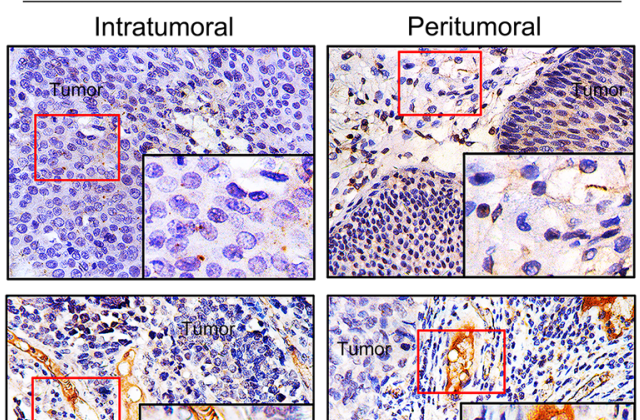

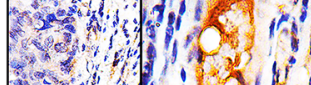

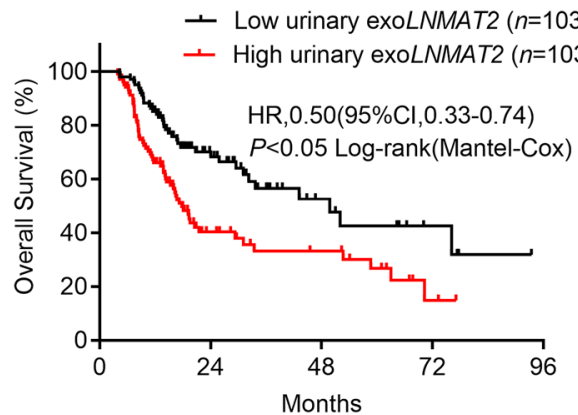
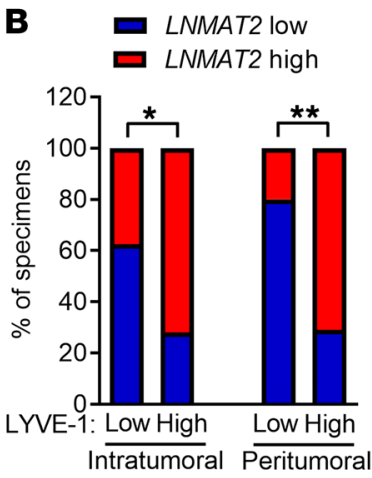

\section{E}

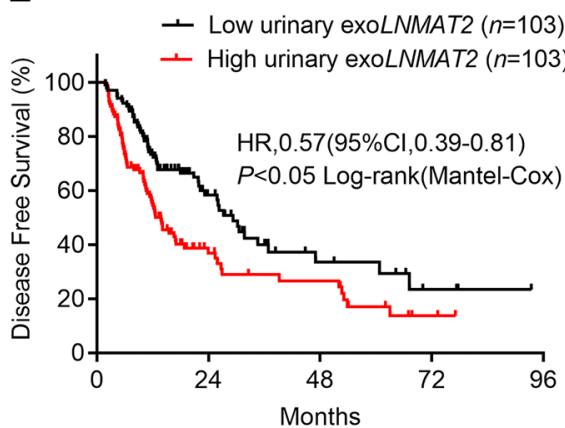

H

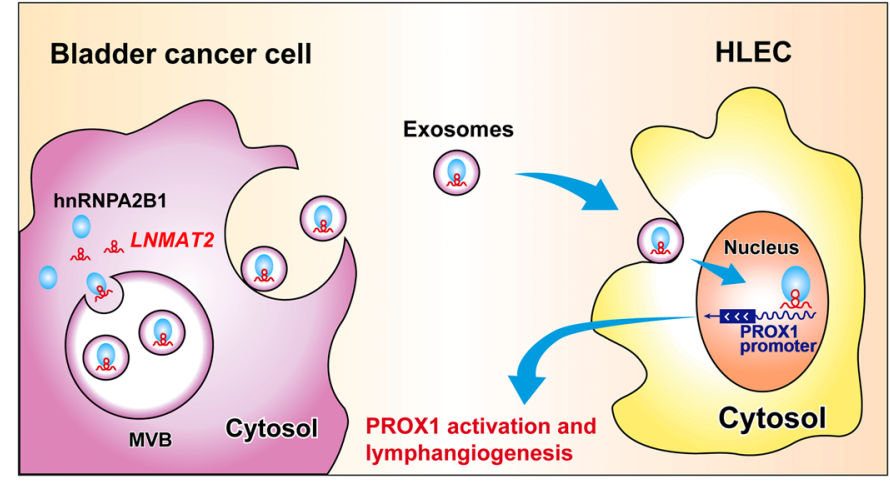

Figure 10. Exosomal LNMAT2 is associated with BCa lymphatic metastasis. Representative images $(\mathbf{A})$ and percentages $(\mathrm{B})$ of $\mathrm{BCa}$ tissues $(n=206)$ with high and low LYVE-1 levels in the intratumoral and peritumoral lymphatic vessels in patients with different expression of LNMAT2. Scale bars: $50 \mu \mathrm{m}$. Statistical significance was assessed by $\chi^{2}$ test. (C) qRT-PCR analysis of LNMAT2 expression in a 206-patient cohort of urinary-EXO from BCa patients with or without LN metastasis. CAPDH was used as an internal control. Groups were compared using the nonparametric Mann-Whitney $U$ test. Kaplan-Meier curves of $0 S$ (D) and DFS (E) of patients with BCa according to the relative urinary exosomal LNMAT2 expression. The median expression was used as the cut-off value $(n=206)$. ROC curve analyses for evaluating the diagnostic potential of urinary exosomal LNMAT2 for BCa (F) and LN metastasis (G). (H) Proposed model of BCa cellsecreted exosomal LNMAT2-mediated PROX1 activation in HLECs for promoting lymphangiogenesis and LN metastasis. ${ }^{*} P<0.05 ;{ }^{* *} P<0.01$. 
ically to LNMAT2 through a specific sequence on 1930-1960 nt of LNMAT2 and directs its packaging into exosomes. The identification of hnRNPA2B1 for exporting LNMAT2 in exosomes may provide unique strategies for eliminating $L N M A T 2$ in the TME and for blocking exosomal LNMAT2-mediated BCa LN metastasis.

Another important finding in the present study was that cancer-secreted exosomal LNMAT2 upregulated PROX1 expression epigenetically in HLECs. PROX1 is the key transcription factor driving HLEC fate and specification by regulating the expression of various lymphatic-specific proteins, including VEGFR3, LYVE-1, and podoplanin $(9,46)$. PROX1 overexpression controls lymphangiogenesis by inducing HLEC proliferation and migration $(47,48)$. Importantly, PROX1 depletion in mouse models causes lymphatic defects that lead to mortality (9). Although several studies have indicated the essential role of PROX1 in lymphangiogenesis, cancerinduced $P R O X 1$ transcription in HLECs remains unknown. Herein, we demonstrated that BCa cell-secreted exosomal LNMAT2 upregulated PROX1 expression in HLECs by forming a DNA-RNA triplex with the binding site of the PROX1 promoter. PROX1 downregulation abolished the prolymphangiogenesis effect induced by exosomal LNMAT2. Our findings uncovered what we believe is a novel molecular mechanism underlying the cancer-secreted exosomal lncRNA-mediated PROX1 overexpression in HLECs, resulting in lymphangiogenesis and LN metastasis, which expanded the current knowledge on PROX1 regulation in HLECs.

In summary, our findings provided evidence of a VEGF-Cindependent LN metastasis mechanism in which BCa cell-secreted exosomal LNMAT2 promoted lymphangiogenesis and LN metastasis by transcriptionally upregulating PROX1 in HLECs. We also found that LNMAT2 was overexpressed in both the urinary-EXO and serum-EXO of BCa patients, which positively correlated with both intratumoral and peritumoral lymphangiogenesis, and was clinically relevant to BCa LN metastasis. Our study not only identifies a crucial mechanism of exosomal lncRNAmediated intercellular communication from BCa cells to the TME to provoke LN metastasis, but also develops a potential noninvasive diagnostic approach and therapeutic strategy for patients with BCa with LN metastasis.

\section{Methods}

Clinical samples and study approval. A total of 266 pairs of tumor tissues and NATs from patients with BCa who underwent surgery was obtained at Sun Yat-sen Memorial Hospital (Cohort 1). Urine and blood samples were obtained from another 206 patients with BCa and 120 healthy participants (Cohort 2). In both cohorts, patients were eligible if they had pathologically confirmed BCa. The clinical features of the patients are summarized in Supplemental Table 1 and Supplemental Table 7. All experiments were conducted with the approval of the Committees for Ethical Review of Research involving Human Subjects at Sun Yat-sen University. Written informed consent was obtained from all participants prior to sample collection.

Cell lines and cell culture. The human BCa cell lines UM-UC-3 (CRL-1749), 5637 (HTB-9), and T24 (HTB-4), and the immortalized normal human urothelial cell line SV-HUC-1 (CRL-9520) were purchased from American Type Culture Collection. UM-UC-3 and T24 cells were cultured in DMEM (Gibco) supplemented with 10\% FBS. The 5637 and SV-HUC-1 cells were cultured in RPMI 1640 medium
(Gibco) supplemented with 10\% FBS and F-12K medium (HyClone) supplemented with 10\% FBS, respectively. HLECs (catalog 2500) were purchased from ScienCell Research Laboratories and cultured in endothelial cell medium (ECM) (ScienCell Research Laboratories) supplemented with $5 \%$ FBS. The cells were cultured in a humidified incubator with $5 \% \mathrm{CO}_{2}$ at $37^{\circ} \mathrm{C}$.

Mouse popliteal lymphatic metastasis model. BALB/c nude mice (4-5 weeks old, 18-20 g) were purchased from the Experimental Animal Center, Sun Yat-sen University (Guangzhou, China) and were used for the lymphatic metastasis model. All experimental procedures were conducted with the approval of the Sun Yat-sen University IACUC. Luciferase-labeled UM-UC-3 cells $\left(5 \times 10^{6}\right)$ were inoculated into the footpads of the mice. Then, the mice were randomly divided into 3 groups ( $n=12$ or 16 per group) and injected intratumorally with PBS, UM-UC-3-EXO ${ }_{\text {Vector }}$, or UM-UC-3-EXO LNMAT2 $_{2}(20 \mu \mathrm{g} /$ dose $)$ every 3 days. Lymphatic metastasis was analyzed using a PerkinElmer IVIS Spectrum In Vivo Imaging System. The footpad tumors and popliteal LNs were excised when the tumors were $200 \mathrm{~mm}^{3}$ ( $\mathrm{LN}$ volume $\left(\mathrm{mm}^{3}\right)$ $=\left(\right.$ length $[\mathrm{mm}] \times$ width $\left.\left.[\mathrm{mm}]^{2}\right) / 2\right)$. Serial sections of primary tumors and popliteal LNs were analyzed by ISH and IHC. The sections were visualized with a Nikon Eclipse Ti microscope. For survival analysis, the mice were observed until death or were sacrificed by cervical dislocation 80 days after the first injection of PBS or exosomes.

RNA pull-down and RIP assays. The LNMAT2-binding proteins were examined using RNA pull-down assays according to the instructions of the Pierce Magnetic RNA-Protein Pull-Down Kit (Thermo Fisher Scientific). Biotinylated LNMAT2 and antisense sequences were synthesized using a TranscriptAid T7 High Yield Transcription Kit (Thermo Fisher Scientific). The nuclear fraction obtained using a NE-PER Nuclear Protein Extraction Kit (Thermo Fisher Scientific) was incubated overnight with biotinylated LNMAT2, followed by precipitation with streptavidin magnetic beads. The retrieved protein was eluted from the RNA-protein complex and analyzed by immunoblotting or silver staining, followed by MS analysis with a MALDI-TOF instrument (Bruker Daltonics).

The RIP assays were performed using an EZ-Magna RIP kit (Millipore). Lysates of $1 \times 10^{7} \mathrm{BCa}$ cells obtained using complete RIP lysis buffer were immunoprecipitated with RIP buffer containing anti-hnRNPA2B1 antibody-conjugated magnetic beads (Abcam). The precipitated RNAs were analyzed by qRT-PCR. Mouse IgG and U1 RNA were used as the negative and nonspecific controls, respectively.

FRET and CDspectroscopy. For FRET assays, 5-carboxytetramethylrhodamine-labeled (TAMRA-labeled) TFO and 5-carboxyfluoresceinlabeled (FAM-labeled) TTSs were generated and mixed in binding buffer (20 mM HEPES [pH 7.5], $10 \mathrm{mM} \mathrm{MgCl}, 50 \mathrm{mM}$ sodium acetate) in a ratio of 1:5 (500 $\mathrm{nM} \mathrm{TTS} / 2500 \mathrm{nM} \mathrm{TFO})$. The mixtures were incubated at $55^{\circ} \mathrm{C}$ for 10 minutes, followed by a 10-hour incubation at $37^{\circ} \mathrm{C}$. The fluorescence wavelengths between 480 and $690 \mathrm{~nm}$ were measured using a Molecular Device M5 Plate Reader.

For the CD spectroscopy, a 1:1 mixture of TFO $(2.2 \mu \mathrm{M})$ and TTS oligos $(2.2 \mu \mathrm{M})$ in binding buffer (20 mM HEPES [pH 7.5], $10 \mathrm{mM}$ $\mathrm{MgCl}_{2}, 50 \mathrm{mM}$ sodium acetate) was equilibrated at $30^{\circ} \mathrm{C}$ for 1 hour. Control ssRNA/PROX1 TTS and FENDRR TFO/PITX2 TTS were used as the negative and positive controls, respectively. The measurements were performed on a Chirascan spectrometer (Applied Photophysics). The oligos used in the FRET and CD spectroscopy are listed in Supplemental Table 5. 
Data availability. The NGS data for this study (GSE106534, GSE106637) are available on the National Center for Biotechnology Information website (https://www.ncbi.nlm.nih.gov/geo/query/acc. cgi?acc=GSE106534). All relevant data within the scope of the paper are publicly available.

Bioinformatics analysis. The hnRNPA2B1 binding motif enrichment in RNAs was obtained via POSTAR2. The LNMAT2 secondary structure was predicted using RNAalifold. The LNMAT2 binding motifs in the PROX1 promoter and the binding sequences in LNMAT2 were predicted by LongTarget.

Statistics. Statistical differences between groups were evaluated using SPSS v.13.0 (SPSS Inc.). Data were considered statistically significant if $P$ was less than 0.05. All experiments were performed in triplicate, and quantitative data are presented as the mean \pm SD. Statistical significance for samples with nonnormal distribution was identified using the Mann-Whitney $U$ test, 2-tailed Student's $t$ test or 1-way ANOVA was applied for parametric variables, and the $\chi^{2}$ test was applied for nonparametric variables. OS and DFS were evaluated using the Kaplan-Meier method.

Study approval. The use of human BCa tissue specimens was evaluated and approved by the Ethical Committee of Sun Yat-sen Memorial Hospital, Sun Yat-sen University, and written informed consent was obtained from all participants or their appropriate surrogates. All animal studies were conducted with approval from the Sun Yat-sen University Institutional Animal Care and Use Committee and were performed in accordance with established guidelines.

\section{Author contributions}

$\mathrm{CC}, \mathrm{JH}, \mathrm{RC}$, and TL conceived, designed, and directed the study. $\mathrm{Y}$ Li, Y Luo, WH, and YZ performed the in vitro and in vivo experiments and data analyses. YK, YZ, and HL performed the clinical data analyses. WH and GZ performed the ISH and IHC experiments. CC, JL, and Y Luo wrote and critically reviewed the man- uscript. All authors read and approved the final manuscript. The order of the co-first authors was assigned based on the relative contributions of these individuals.

\section{Acknowledgments}

The authors thank JX Zhang of the Department of Medical Statistics and Epidemiology, School of Public Health, Sun Yat-sen University, for statistical advice and research comments. This study was funded by the National Key Research and Development Program of China (grant no. 2018YFA0902803); the National Natural Science Foundation of China (grant nos. 81825016, 81802530, 81830082, 81772719, 81772728, 91740119); Guangdong Medical Research Foundation (grant no. A2018330); Medical Scientific Research Foundation of Guangdong Province (grant no. A201947); Science and Technology Program of Guangzhou (grant nos. 201604020156, 201604020177, 201707010116, 2017B020227007); National Natural Science Foundation of Guangdong (grant nos. 2018A030313564, 2018A030310250, 2016A030313321); Yixian Youth project of Sun Yat-sen Memorial Hospital (grant no. YXQH201812); The Key Areas Research and Development Program of Guangdong (grant no. 2018B010109006); Guangdong Province Higher Vocational Colleges \& Schools Pearl River Scholar Funded Scheme (to TL).

Address correspondence to: Tianxin Lin and Jian Huang, Department of Urology, Sun Yat-sen Memorial Hospital, 107 Yanjiangxi Road, Yuexiu District, Guangzhou, Guangdong, 510120, China. Phone: 86.20.34070447; Email: lintx@mail.sysu.edu.cn, urolhj@sina.com. Or to: Rufu Chen, Department of General Surgery, Guangdong Provincial People's Hospital, Guangdong Academy of Medical Sciences, Guangzhou, Guangdong, 510080, China. Phone: 86.20.18922182828; Email: chenrf63@163.com.
1. Bray F, Ferlay J, Soerjomataram I, Siegel RL, Torre LA, Jemal A. Global cancer statistics 2018: GLOBOCAN estimates of incidence and mortality worldwide for 36 cancers in 185 countries. $C A$ Cancer J Clin. 2018;68(6):394-424.

2. Hautmann RE, de Petriconi RC, Pfeiffer C, Volkmer BG. Radical cystectomy for urothelial carcinoma of the bladder without neoadjuvant or adjuvant therapy: long-term results in 1100 patients. Eur Urol. 2012;61(5):1039-1047.

3. He W, et al. Long noncoding RNA BLACAT2 promotes bladder cancer-associated lymphangiogenesis and lymphatic metastasis. JClin Invest. 2018;128(2):861-875.

4. Chen C, et al. LNMAT1 promotes lymphatic metastasis of bladder cancer via CCL2 dependent macrophage recruitment. Nat Commun. 2018;9(1):3826.

5. Zu X, Tang Z, Li Y, Gao N, Ding J, Qi L. Vascular endothelial growth factor-C expression in bladder transitional cell cancer and its relationship to lymph node metastasis. BJU Int 2006;98(5):1090-1093.

6. Suzuki K, Morita T, Tokue A. Vascular endothelial growth factor-C (VEGF-C) expression predicts lymph node metastasis of transitional cell carcinoma of the bladder. Int JUrol. 2005;12(2):152-158.
7. Saharinen P, Tammela T, Karkkainen MJ, Alitalo K. Lymphatic vasculature: development, molecular regulation and role in tumor metastasis and inflammation. Trends Immunol. 2004;25(7):387-395.

8. Plate K. From angiogenesis to lymphangiogenesis. Nat Med. 2001;7(2):151-152.

9. Wigle JT, et al. An essential role for Prox1 in the induction of the lymphatic endothelial cell phenotype. EMBO J. 2002;21(7):1505-1513.

10. Flister MJ, et al. Inflammation induces lymphangiogenesis through up-regulation of VEGFR-3 mediated by NF-kappaB and Prox1. Blood. 2010;115(2):418-429.

11. Zhang ZG, Buller B, Chopp M. Exosomes beyond stem cells for restorative therapy in stroke and neurological injury. Nat Rev Neurol. 2019;15(4):193-203.

12. Xu R, Rai A, Chen M, Suwakulsiri W, Greening DW, Simpson RJ. Extracellular vesicles in cancer - implications for future improvements in cancer care. Nat Rev Clin Oncol. 2018;15(10):617-638.

13. Fang JH, et al. Hepatoma cell-secreted exosomal microRNA-103 increases vascular permeability and promotes metastasis by targeting junction proteins. Hepatology. 2018;68(4):1459-1475.

14. Pan Y, et al. Adipocyte-secreted exosomal microRNA-34a inhibits M2 macrophage polariza- tion to promote obesity-induced adipose inflammation. J Clin Invest. 2019;129(2):834-849.

15. Cheng $\mathrm{M}$, et al. Circulating myocardial microRNAs from infarcted hearts are carried in exosomes and mobilise bone marrow progenitor cells. Nat Commun. 2019;10(1):959.

16. Zhang $\mathrm{H}$, et al. Exosome-delivered EGFR regulates liver microenvironment to promote gastric cancer liver metastasis. Nat Commun. 2017;8:15016.

17. Zeng Z, et al. Cancer-derived exosomal miR-253 p promotes pre-metastatic niche formation by inducing vascular permeability and angiogenesis. Nat Commun. 2018;9(1):5395.

18. Fang T, et al. Tumor-derived exosomal miR-1247$3 p$ induces cancer-associated fibroblast activation to foster lung metastasis of liver cancer. Nat Commun. 2018;9(1):191.

19. Mathieu M, Martin-Jaular L, Lavieu G, Théry C. Specificities of secretion and uptake of exosomes and other extracellular vesicles for cell-to-cell communication. Nat Cell Biol. 2019;21(1):9-17.

20. Ji H, et al. TNFR1 mediates TNF- $\alpha$-induced tumour lymphangiogenesis and metastasis by modulating VEGF-C-VEGFR3 signalling. Nat Commun. 2014;5:4944.

21. Chen F, et al. Extracellular vesicle-packaged 
HIF-1 $\alpha$-stabilizing IncRNA from tumour-associated macrophages regulates aerobic glycolysis of breast cancer cells. Nat Cell Biol. 2019;21(4):498-510.

22. Karaman S, Detmar M. Mechanisms of lymphatic metastasis. JClin Invest. 2014;124(3):922-928.

23. Seok Y, et al. Frequency of lymph node metastasis according to the size of tumors in resected pulmonary adenocarcinoma with a size of $30 \mathrm{~mm}$ or smaller. J Thorac Oncol. 2014;9(6):818-824.

24. Liu YY, et al. Does a higher cutoff value of lymph node retrieval substantially improve survival in patients with advanced gastric cancer?-time to embrace a new digit. Oncologist. 2017;22(1):97-106.

25. Rault-Petit B, et al. Current management and predictive factors of lymph node metastasis of appendix neuroendocrine tumors: a national study from the french group of endocrine tumors (GTE). Ann Surg. 2019;270(1):165-171.

26. Moran-Jones K, et al. hnRNP A2, a potential ssDNA/RNA molecular adapter at the telomere. Nucleic Acids Res. 2005;33(2):486-496.

27. Zhu Y, et al. POSTAR2: deciphering the post-transcriptional regulatory logics. Nucleic Acids Res. 2019;47(D1):D203-D211.

28. Hu B, Yang YT, Huang Y, Zhu Y, Lu ZJ. POSTAR: a platform for exploring post-transcriptional regulation coordinated by RNA-binding proteins. Nucleic Acids Res. 2017;45(D1):D104-D114.

29. Villarroya-Beltri C, et al. Sumoylated hnRNPA2B1 controls the sorting of miRNAs into exosomes through binding to specific motifs. Nat Commun. 2013;4:2980.

30. Shurtleff MJ, Temoche-Diaz MM, Karfilis KV, Ri $\mathrm{S}$, Schekman R. Y-box protein 1 is required to sort microRNAs into exosomes in cells and in a cell- free reaction. Elife. 2016;5:e19276.

31. Park KJ, et al. Prospero homeobox 1 mediates the progression of gastric cancer by inducing tumor cell proliferation and lymphangiogenesis. Gastric Cancer. 2017;20(1):104-115.

32. Cho H, et al. YAP and TAZ negatively regulate Prox1 during developmental and pathologic lymphangiogenesis. Circ Res. 2019;124(2):225-242.

33. He S, Zhang H, Liu H, Zhu H. LongTarget: a tool to predict lncRNA DNA-binding motifs and binding sites via Hoogsteen base-pairing analysis. Bioinformatics. 2015;31(2):178-186.

34. Grote P, Herrmann BG. The long non-coding RNA Fendrr links epigenetic control mechanisms to gene regulatory networks in mammalian embryogenesis. RNA Biol. 2013;10(10):1579-1585.

35. Tchurikov NA, et al. Hot spots of DNA double-strand breaks and genomic contacts of human rDNA units are involved in epigenetic regulation. J Mol Cell Biol. 2015;7(4):366-382.

36. Tchurikov NA, et al. DNA double-strand breaks coupled with PARP1 and HNRNPA2B1 binding sites flank coordinately expressed domains in human chromosomes. PLoS Genet. 2013;9(4):e1003429.

37. Zhan Y, et al. Expression signatures of exosomal long non-coding RNAs in urine serve as novel non-invasive biomarkers for diagnosis and recurrence prediction of bladder cancer. Mol Cancer. 2018;17(1):142.

38. Alitalo K, Tammela T, Petrova TV. Lymphangiogenesis in development and human disease. Nature. 2005;438(7070):946-953.

39. Xue M, et al. Hypoxic exosomes facilitate bladder tumor growth and development through trans- ferring long non-coding RNA-UCA1. Mol Cancer. 2017;16(1):143.

40. Xu H, et al. Exosome-transmitted PSMA3 and PSMA3-AS1 promote proteasome inhibitor resistance in multiple myeloma. Clin Cancer Res. 2019;25(6):1923-1935.

41. Ren J, et al. Carcinoma-associated fibroblasts promote the stemness and chemoresistance of colorectal cancer by transferring exosomal lncRNA H19. Theranostics. 2018;8(14):3932-3948.

42. Fernández MI, et al. Prognostic implications of lymphangiogenesis in muscle-invasive transitional cell carcinoma of the bladder. Eur Urol. 2008;53(3):571-578.

43. Kluth LA, et al. Prognostic and prediction tools in bladder cancer: a comprehensive review of the literature. Eur Urol. 2015;68(2):238-253.

44. Fluiter K, Mook OR, Baas F. The therapeutic potential of LNA-modified siRNAs: reduction of off-target effects by chemical modification of the siRNA sequence. Methods Mol Biol. 2009;487:189-203.

45. $\mathrm{Qu} \mathrm{L}$, et al. Exosome-transmitted lncARSR promotes sunitinib resistance in renal cancer by acting as a competing endogenous RNA. Cancer Cell. 2016;29(5):653-668.

46. Lee S, et al. Prox1 physically and functionally interacts with COUP-TFII to specify lymphatic endothelial cell fate. Blood. 2009;113(8):1856-1859.

47. Cha B, et al. Complementary Wnt sources regulate lymphatic vascular development via PROX1-dependent Wnt/ $\beta$-catenin signaling. Cell Rep. 2018;25(3):571-584.e5.

48. Wong BW, et al. The role of fatty acid $\beta$-oxidation in lymphangiogenesis. Nature. 2017;542(7639):49-54. 